\title{
Age At Menarche, Age At Menopause, Reproductive Years and Risk of Fatal Stroke Occurrence Among Chinese Women: The Guangzhou Biobank Cohort Study
}

\section{Zhi-bing Hu}

Guangzhou Twelfth People's Hospital

\section{Ze-xiong Lu}

Sanya Central Hospital

Feng Zhu ( $\nabla$ chifengzhu@hotmail.com )

Guangzhou Twelfth People's Hospital

\section{Research Article}

Keywords: Stroke, Ischaemic, Menarche, Menopause, Reproductive

Posted Date: August 27th, 2021

DOI: https://doi.org/10.21203/rs.3.rs-763575/v1

License: (1) (1) This work is licensed under a Creative Commons Attribution 4.0 International License. Read Full License

Version of Record: A version of this preprint was published at BMC Women's Health on December 1st, 2021. See the published version at https://doi.org/10.1186/s12905-021-01579-9. 


\section{Abstract \\ Background}

The relationship between women's reproductive characteristics and stroke events is unclear. We aimed to investigate age at menarche, age at menopause and number of reproductive years in relation to fatal stroke occurrence in the Guangzhou Biobank Cohort Study.

\section{Methods}

In total, 16504 postmenopausal women without stroke, heart disease or a cancer history at baseline were included and followed up for a median of 12.0 years. After review of available records, 222 stroke deaths were recorded. Cox proportional hazards regression was used to assess the associations between the risk of fatal stroke occurrence and age at menarche, age at menopause and number of reproductive years.

\section{Results}

In the whole cohort, compared with those aged 15 years at menarche, women aged 17 years at menarche had an increased risk for fatal stroke (adjusted hazard ratio [aHR] $=1.83,95 \%$ confidence interval $(\mathrm{Cl})$ $1.10-3.05)$ and fatal haemorrhagic stroke $(\mathrm{HR}=2.65,95 \% \mathrm{Cl} 1.14-6.18)$, and women aged $\geq 18$ years at menarche had an increased risk for fatal stroke $(H R=1.66,95 \% \mathrm{Cl} 1.03-2.70)$ and fatal ischaemic stroke $(\mathrm{HR}=2.01,95 \% \mathrm{Cl} 1.01-3.99)$. Among postmenopausal women born before 1940, women aged $<43$ years at menopause had an increased risk for fatal stroke $(H R=1.97,95 \% \mathrm{Cl} 1.05-3.69)$ compared with those aged 51-52 years at menopause. Additionally, in the whole cohort, women with $\leq 28$ reproductive years had an increased risk for fatal stroke $(H R=1.91,95 \% \mathrm{Cl} 1.28-2.86)$ and fatal ischaemic stroke (HR $=2.26,95 \% \mathrm{Cl} 1.26-4.05)$ compared with those with 32-34 reproductive years; postmenopausal women born before 1940 had a similar risk for fatal stroke and fatal ischaemic stroke.

\section{Conclusions}

Older age at menarche, younger age at menopause and fewer reproductive ages were related to an increased risk of fatal stroke in postmenopausal women.

\section{Background}

In China, the life expectancy of women is 79.9 years, which is approximately 6.5 years longer than that of men [1]. Stroke is a leading cause of death in China [2] and is considered to be a sexually dimorphic disease [3]. Compared with men, women more easily suffer from cerebrovascular disease [4], have a higher stroke incidence [5] and have poorer functional outcomes for intravenous thrombolysis (IVT)treated ischaemic stroke [6]. One study revealed that sex had a modifying effect on mortality in diabetic 
patients with first-ever ischaemic stroke, showing an increased risk of long-term mortality among women [7].

Menarche is the beginning, and menopause is the end of a woman's reproductive timeline, and the reproductive years, which is the interval between menarche and menopause, is a woman's natural reproductive window. Recent studies have reported that early menarche is associated with an increased mortality or incidence risk of stroke [8-15], though there are conflicting reports[12, 16-23]. Additionally, a U-shaped association [24] and an increased risk of stroke incidence have been found in those with late menarche $[19,25]$. However, early menarche showed a $16 \%$ lower but an $18 \%$ higher risk of stroke among women born during the 1920s-1940s and women born in the 1950s, respectively, and a U-shaped association among women born in the 1960s-1970s [14]. Fewer reproductive years was also associated with stroke mortality or incidence [10,12, 25-27], though there were inconsistencies in results $[8,19]$. Thus, these previous studies on women showed a mixed relationship between reproductive characteristics and stroke events. Here, we aimed to systematically assess the associations between age at menarche, age at menopause, and number of reproductive years and the risk of fatal stroke occurrence in healthy postmenopausal Chinese women.

\section{Methods}

\section{Data source and participants}

All GBCS participants were recruited from a population of permanent residents aged 50 years or older in Guangzhou in southern China. Details of the GBCS have been reported previously [28]. The baseline (from September $1^{\text {st }}, 2003$, to February $28^{\text {th }}, 2008$ ) included a face-to-face computer-assisted interview by trained nurses on lifestyle, family and personal medical history; assessments of anthropometrics and blood pressure; and a series of laboratory tests, including fasting plasma glucose and lipids. Physical activity was assessed by means of the Chinese version of the International Physical Activity Questionnaire (IPAQ) and was classified into inactive, moderately active and physically active [29].

\section{Exposure indicators}

The ages at menarche and menopause were recorded in years (per the Gregorian calendar and related interpretation of age) and rounded during data collection to the nearest year (e.g., 13 years represents the onset of menarche from 12 years 6 months to 13 years 5 months) [30]. The number of reproductive years was generated by subtracting age at menarche from age at menopause. Data on each woman's history of oral contraceptive $(\mathrm{OC})$ use, hormone replacement therapy, hysterectomy and ovarian or breast surgery were also recorded.

\section{Exposure indicators}

Information on underlying causes of death up to December $31^{\text {st }}, 2017$, was obtained mostly via record linkage with the Guangzhou Centres for Disease Control and Prevention (GZCDC). Because there was no 
other information for stroke severity, infarct volume, lesion site or infectious complications, fatal stroke occurrence was chosen as the primary outcome of this study. Death causes were coded according to the $10^{\text {th }}$ revision of the International Classification of Diseases (ICD) as follows: 160 169 for stroke; 160.0 162.9 and 169.0 169.2 for haemorrhagic stroke; 163.0 163.9 and I69.3 for ischaemic stroke; and the other codes for unclassified stroke. When the death certificates were not issued by medical institutions, the causes were verified by GZCDC as part of their quality assurance programmed by cross-checking past medical history and conducting verbal autopsy by 5 senior clinicians from Guangzhou Twelfth People's Hospital, the Universities of Hong Kong, China, and Birmingham, UK.

To examine the extent to which baseline factors explained the associations of stroke, ischaemic stroke and haemorrhagic stroke, we included the factors in different models. Model 1 was a crude hazard ratio model without adjustment for any confounders. Model 2 contained a multivariate adjustment for factors including age, diabetes, hypertension, dyslipidaemia, smoking, alcohol consumption, physical activity, body mass index, self-rated health, education, job, family income, number of children and oral contraceptive pill use.

\section{Statistical analysis}

Cox proportional hazards regression was used to analyse the association between age at menarche and age at menopause, reproductive years and the risk of fatal stroke. The age at menarche was categorized into 7 age groups: $\leq 12,13,14,15,16,17$, and $\geq 18$ years [14]. The age at menopause was categorized into 5 age groups: $<43,43-47,48-50,51-52$, and $\geq 53$ years [26]. The reproductive years were categorized into 5 age groups: $\leq 28,29-31,32-34,35-37$, and $\geq 38$ years. Fewer reproductive years was defined as the lowest deciles [19]. Covariates included age, diabetes, hypertension, dyslipidaemia, smoking, alcohol consumption, physical activity, body mass index, self-rated health, education, job, family income, number of children and oral contraceptive pill use. Similar to a previous Chinese population study [31], our cohort showed that age at menarche decreased and that age at menopause increased in younger generations. Thus, further analysis was conducted by birth cohort; by smoking status (never smoker with additional adjustments for childhood passive smoking exposure), which is linked to an earlier age at menarche [32] and menopause [33]; and by stroke mortality [34]. A sensitivity analysis was conducted after exclusion of those with removal of the uterus, ovaries, or breast masses (benign or malignant) or those who received hormone replacement therapy. All analyses were performed using STATA (version 14.0; StataCorp LP, College Station, TX, USA). All p values were 2 sides, and statistical significance was defined as $\mathrm{P}<0.05$.

\section{Results}

\section{Baseline characteristics}

In total, 22067 (72.5\%) female participants comprising 20343 postmenopausal women were screened. Among patient exclusions, 244 were lost to follow-up and had an unknown vital status, 157 had a history 
of stroke, 1741 had heart disease or peripheral vascular disease, 363 had cancer, and 1334 had incomplete information on diabetes, hypertension, dyslipidaemia, smoking, alcohol consumption, physical activity, body mass index, self-rated health, education, job, family income, number of children and oral contraceptive pill use. A total of 16504 participants who were free of stroke at baseline were included in this study. After a median follow-up of 12.0 years, 222 stroke deaths ( 95 ischaemic, 82 haemorrhagic and 45 unclassified) were recorded (Fig. 1). Among the postmenopausal women, the mean age was 61.4 (6.8) years, and the age at menarche was 15.1 (2.1) years, with a decreasing trend (from 15.7 (2.1) years among those born before 1940 to 14.9 (2.0) years among those born in 1940 or later); the age at menopause was 49.5 (3.8) years, with an increasing trend (from 48.9 (4.1) years among those born before 1940 to 49.7 (3.6) years among those born in 1940 or later); and the number of reproductive years was 34.3 (4.3) years, with an increasing trend (from 33.2 (4.6) years among those born before 1940 to 34.8 (4.0) years among those born in 1940 or later).

The baseline characteristics of the participants according to age at menarche are presented in Table 1. Compared with those aged 15 years at menarche, those with early menarche $(\leq 12)$ were younger; had lower rates of $\leq$ primary education and manual jobs; had a higher family income, proportion of women with a number of children born $>3$, and a higher number of reproductive years; and had higher proportions of current smokers, alcohol consumers and women with physical activity. Compared with those aged 15 years at menarche, those with late menarche $(\geq 18)$ were older, comprised more current smokers, and were more likely to engage in physical activity; had higher rates of $\leq$ primary education and manual jobs; had a higher family income and proportion of women with a number of children $>3$; had lower rates of hypertension, diabetes, current drinking, and oral contraceptive pill use; and had fewer reproductive years. 
Table 1

Baseline characteristics by age at menarche of participants in the GBCS

\section{Characteristics}

Overall Age at menarche (years)

$\begin{array}{lllllll}\leq 12 & 13 & 14 & 15 & 16 & 17 & \geq 18\end{array}$

Number, $\mathrm{n}$

165041368

61.4

59.0

27.0

27.6

27.8

27.9

$13.7 \quad 12.3$

13.3

13.1

85.7

87.4

87.6

86.8

85.9

85.2

84.2

82.7

BMI $\left(\mathrm{kg} / \mathrm{m}^{2}\right)^{\mathrm{b}}$

23.8

$24.3 \quad 23.9$

23.8

82.7

82.6

83.5

83.0

23.8

23.8

23.8

23.6

Good/very good self-rated health, \% ${ }^{\mathrm{c}}$

Oral contraceptive pill use, $\%$

Current smoker, $\%^{\mathrm{b}}$

Current drinking, $\%^{\mathrm{b}}$

Active physical activity, \% ${ }^{\mathrm{c}}$

Education $\leq$ primary, \%

Manual job, \%

Family income $<30000$

$\mathrm{CNY} /$ year, ${ }^{\mathrm{b}}$

Number of children born $>3, \%$

Age at menopause (years) ${ }^{\mathrm{b}}$

Reproductive years (years) ${ }^{b}$
17.8

1.9

20.6

52.6

49.9

54.5

38.3

21.4

49.5

34.3
$17.6 \quad 18.9$

2.0

$26.4 \quad 23.0$

52.1

53.0

31.5

33.3

43.3

47.1

33.5

34.9

35.0

8.6

10.9

13.2

49.0

49.4

49.6

37.2
36.4
1.5

22.2

52.2

38.6

47.7
19.7

1.7

2.5

1.6

2.3

54.8

38.0

38.0

21.2

$\begin{array}{lllll}35.6 & 34.6 & 33.6 & 32.5 & 30.9\end{array}$

a: $P<0.05$; b: $P<0.01$; c: $P>0.05$; hypertension: systolic blood pressure, $\geq 140 \mathrm{mmHg}$, diastolic blood pressure, $\leq 90 \mathrm{mmHg}$, medication or diagnosed; diabetes: fasting blood glucose $\geq 7$ or medication or diagnosis; dyslipidaemia: total cholesterol $\geq 5.2 \mathrm{mmol} / \mathrm{L}$, triglyceride $\geq 1.7 \mathrm{mmol} / \mathrm{L}$, low density lipoprotein $\geq 3.4 \mathrm{mmol} / \mathrm{L}$, high density lipoprotein $<1.0 \mathrm{mmol} / \mathrm{L}$, medication or diagnosis; BMl: body mass index. 


\section{Menarche In Relation To Fatal Stroke Occurrence}

Menarche in relation to fatal stroke occurrence is presented in Table 2. Compared with those aged 15 years at menarche, those with late menarche at 17 and $\geq 18$ years of age had an increased risk of fatal stroke $(\mathrm{HR}=1.83,95 \% \mathrm{Cl} 1.10-3.05, \mathrm{P}=0.02 ; \mathrm{HR}=1.66,95 \% \mathrm{Cl} 1.03-2.70, \mathrm{P}=0.04)$ in the whole cohort. Similar associations were found for menarche age at 17 years of age $(H R=2.30,95 \% \mathrm{Cl} 1.20-4.39, \mathrm{P}=$ $0.01)$ and 18 years of age $(\mathrm{HR}=2.50,95 \% \mathrm{Cl} 1.37-4.57, \mathrm{P}=0.003)$. Additionally, a U-shaped association was found for those with menarche at age at $\leq 12$ years of age $(\mathrm{HR}=3.68,95 \% \mathrm{Cl} 1.68-8.05, \mathrm{P}=0.001)$, 13 years of age $(\mathrm{HR}=2.11,95 \% \mathrm{Cl} 1.02-4.34, \mathrm{P}=0.04)$ and 14 years of age $(\mathrm{HR}=2.07,95 \% \mathrm{Cl} 1.04, \mathrm{P}=$ $0.001)$ in the cohort of postmenopausal women born before 1940. Late menarche $(\geq 18)$ was associated with an increased risk of fatal ischaemic stroke occurrence in the whole cohort $(\mathrm{HR}=2.01,95 \% \mathrm{Cl} 1.01-$ $3.99, \mathrm{P}=0.04)$ and the cohort of postmenopausal women born before $1940(\mathrm{HR}=2.93,95 \% \mathrm{Cl} 1.26-6.82$, $P=0.01)$. Furthermore, there was an increased risk of fatal haemorrhagic stroke with menarche at 17 years of age $(H R=2.65,95 \% \mathrm{Cl} 1.14-6.18, \mathrm{P}=0.02)$ in the whole cohort and menarche at $\leq 12$ years of age $(\mathrm{HR}=6.16,95 \% \mathrm{Cl} 1.69-22.40, \mathrm{P}=0.006), 14$ years of age $(\mathrm{HR}=3.28,95 \% \mathrm{Cl} 1.00-10.75, \mathrm{P}=0.05)$ and 17 years of age $(\mathrm{HR}=3.26,95 \% \mathrm{Cl} 1.08-9.89, \mathrm{P}=0.04)$ in the cohort of postmenopausal women born before 1940. However, there were no significant associations between fatal stroke, fatal ischaemic stroke or fatal haemorrhagic stroke and menarche in those born in 1940 or later. All these associations were still observed in a sensitivity analysis after exclusions of patients with removal of the uterus, ovaries, or breast masses (benign or malignant) or who received hormone replacement therapy (Table 3). 
Table 2

Association between fatal stroke occurrence and age at menarche in the GBCS

Age at menarche (years)

$\begin{array}{lllllll}\leq 12 & 13 & 14 & 15 & 16 & 17 & \geq 18\end{array}$

Stroke

\begin{tabular}{|c|c|c|c|c|c|c|c|}
\hline Cohort & 1368 & 2662 & 2849 & 2787 & 2667 & 1720 & 2451 \\
\hline $\begin{array}{l}\text { No. of } \\
\text { deaths }\end{array}$ & $14(0.010)$ & $28(0.011)$ & $\begin{array}{l}28 \\
(0.010)\end{array}$ & $\begin{array}{l}25 \\
(0.009)\end{array}$ & $39(0.015)$ & $37(0.022)$ & $\begin{array}{l}51 \\
(0.021)\end{array}$ \\
\hline $\begin{array}{l}\text { Model } 1 \\
(\mathrm{HR}, \\
95 \% \mathrm{Cl})\end{array}$ & $\begin{array}{l}1.22(0.64- \\
2.35), P= \\
0.55\end{array}$ & $\begin{array}{l}1.19 \\
(0.70- \\
2.05), P= \\
0.52\end{array}$ & $\begin{array}{l}1.13 \\
(0.66- \\
1.94), P= \\
0.66\end{array}$ & 1.00 & $\begin{array}{l}1.63 \\
(0.99- \\
2.69), P= \\
0.06\end{array}$ & $\begin{array}{l}2.39 \\
(1.44- \\
3.97), P= \\
0.001\end{array}$ & $\begin{array}{l}2.34 \\
(1.45- \\
3.77), \\
P= \\
0.001\end{array}$ \\
\hline $\begin{array}{l}\text { Model } 2 \\
(\mathrm{HR}, \\
95 \% \mathrm{Cl})\end{array}$ & $\begin{array}{l}1.86(0.96- \\
3.60), P= \\
0.07\end{array}$ & $\begin{array}{l}1.69 \\
(0.98- \\
2.92), P= \\
0.06\end{array}$ & $\begin{array}{l}1.41 \\
(0.82- \\
2.42), P= \\
0.22\end{array}$ & 1.00 & $\begin{array}{l}1.41 \\
(0.85- \\
2.34), P= \\
0.18\end{array}$ & $\begin{array}{l}1.83 \\
(1.10- \\
3.05), P= \\
0.02\end{array}$ & $\begin{array}{l}1.66 \\
(1.03- \\
2.70) \\
P= \\
0.04\end{array}$ \\
\hline $\begin{array}{l}\text { Order } \\
\text { cohort }^{\$}\end{array}$ & 261 & 511 & 669 & 898 & 887 & 677 & 1035 \\
\hline $\begin{array}{l}\text { No. of } \\
\text { deaths }\end{array}$ & $12(0.046)$ & $16(0.031)$ & $\begin{array}{l}20 \\
(0.030)\end{array}$ & $\begin{array}{l}14 \\
(0.016)\end{array}$ & $27(0.030)$ & $28(0.042)$ & $\begin{array}{l}47 \\
(0.045)\end{array}$ \\
\hline $\begin{array}{l}\text { Model } 1 \\
(\mathrm{HR}, \\
95 \% \mathrm{Cl})\end{array}$ & $\begin{array}{l}3.01(1.39- \\
6.50), P= \\
0.005\end{array}$ & $\begin{array}{l}1.96 \\
(0.96- \\
4.03), P= \\
0.07\end{array}$ & $\begin{array}{l}1.90 \\
(0.96- \\
3.77), P= \\
0.07\end{array}$ & 1.00 & $\begin{array}{l}1.92 \\
(1.01- \\
3.66), P= \\
0.05\end{array}$ & $\begin{array}{l}2.63(1.39- \\
5.00), P= \\
0.003\end{array}$ & $\begin{array}{l}3.04 \\
(1.67- \\
5.51), \\
P< \\
0.001\end{array}$ \\
\hline $\begin{array}{l}\text { Model } 2 \\
(\mathrm{HR}, \\
95 \% \mathrm{Cl})\end{array}$ & $\begin{array}{l}3.68(1.68- \\
8.05), P= \\
0.001\end{array}$ & $\begin{array}{l}2.11 \\
(1.02- \\
4.34), P= \\
0.04\end{array}$ & $\begin{array}{l}2.07 \\
(1.04- \\
4.11), P= \\
0.04\end{array}$ & 1.00 & $\begin{array}{l}1.69 \\
(0.89- \\
3.24), P= \\
0.11\end{array}$ & $\begin{array}{l}2.30 \\
(1.20- \\
4.39), P= \\
0.01\end{array}$ & $\begin{array}{l}2.50 \\
(1.37- \\
4.57), \\
P= \\
0.003\end{array}$ \\
\hline $\begin{array}{l}\text { Younger } \\
\text { cohort }^{\&}\end{array}$ & 1107 & 2151 & 2180 & 1889 & 1780 & 1043 & 1416 \\
\hline $\begin{array}{l}\text { No. of } \\
\text { deaths }\end{array}$ & $2(0.002)$ & $12(0.006)$ & $8(0.004)$ & $\begin{array}{l}11 \\
(0.006)\end{array}$ & $12(0.007)$ & $9(0.009)$ & $\begin{array}{l}4 \\
(0.003)\end{array}$ \\
\hline
\end{tabular}

\$: Postmenopausal women born before 1940 were analysed; \&:Postmenopausal women born in 1940 or later were analysed; model 1: a crude hazard ratio model without adjustments; model 2: a multivariate adjusted model including age, diabetes, hypertension, dyslipidaemia, smoking, alcohol drinking, physical activity, body mass index, self-rated health, education, job, family income, number of children and oral contraceptive pill use. 


\begin{tabular}{|c|c|c|c|c|c|c|c|}
\hline & \multicolumn{7}{|c|}{ Age at menarche (years) } \\
\hline & $\leq 12$ & 13 & 14 & 15 & 16 & 17 & $\geq 18$ \\
\hline $\begin{array}{l}\text { Model } 1 \\
(\mathrm{HR}, \\
95 \% \mathrm{Cl})\end{array}$ & $\begin{array}{l}0.33(0.07- \\
1.50), P= \\
0.15\end{array}$ & $\begin{array}{l}0.97 \\
(0.43- \\
2.21), P= \\
0.95\end{array}$ & $\begin{array}{l}0.65 \\
(0.26- \\
1.63), P= \\
0.36\end{array}$ & 1.00 & $\begin{array}{l}1.17 \\
(0.52- \\
2.65), P= \\
0.71\end{array}$ & $\begin{array}{l}1.49 \\
(0.62- \\
3.59), P= \\
0.38\end{array}$ & $\begin{array}{l}0.48 \\
(0.15- \\
1.51) \\
P= \\
0.21\end{array}$ \\
\hline $\begin{array}{l}\text { Model } 2 \\
(\mathrm{HR}, \\
95 \% \mathrm{Cl})\end{array}$ & $\begin{array}{l}0.37(0.08- \\
1.69), P= \\
0.20\end{array}$ & $\begin{array}{l}1.14 \\
(0.50- \\
2.60), P= \\
0.76\end{array}$ & $\begin{array}{l}0.70 \\
(0.28- \\
1.76), P= \\
0.45\end{array}$ & 1.00 & $\begin{array}{l}1.14 \\
(0.50- \\
2.61), P= \\
0.75\end{array}$ & $\begin{array}{l}1.35 \\
(0.56- \\
3.30), P= \\
0.51\end{array}$ & $\begin{array}{l}0.42 \\
(0.13- \\
1.34) \\
P= \\
0.14\end{array}$ \\
\hline \multicolumn{8}{|c|}{ Ischaemic stroke } \\
\hline Cohort & 1357 & 2651 & 2829 & 2774 & 2640 & 1698 & 2428 \\
\hline $\begin{array}{l}\text { No. of } \\
\text { deaths }\end{array}$ & $3(0.002)$ & $17(0.006)$ & $8(0.003)$ & $\begin{array}{l}12 \\
(0.004)\end{array}$ & $12(0.004)$ & $15(0.009)$ & $\begin{array}{l}28 \\
(0.012)\end{array}$ \\
\hline $\begin{array}{l}\text { Model } 1 \\
(\mathrm{HR}, \\
95 \% \mathrm{Cl})\end{array}$ & $\begin{array}{l}0.55(0.15- \\
1.94), P= \\
0.35\end{array}$ & $\begin{array}{l}1.51 \\
(0.72- \\
3.16), P= \\
0.28\end{array}$ & $\begin{array}{l}0.67 \\
(0.28- \\
1.65), P= \\
0.39\end{array}$ & 1.00 & $\begin{array}{l}1.05 \\
(0.47- \\
2.33), P= \\
0.91\end{array}$ & $\begin{array}{l}2.03 \\
(0.95- \\
4.33), P= \\
0.07\end{array}$ & $\begin{array}{l}2.68 \\
(1.37- \\
5.28) \\
P= \\
0.004\end{array}$ \\
\hline $\begin{array}{l}\text { Model } 2 \\
(\mathrm{HR}, \\
95 \% \mathrm{Cl})\end{array}$ & $\begin{array}{l}0.78(0.22- \\
2.79), P= \\
0.70\end{array}$ & $\begin{array}{l}2.10 \\
(0.99- \\
4.45) \\
P=0.05\end{array}$ & $\begin{array}{l}0.80 \\
(0.32- \\
1.96), P= \\
0.62\end{array}$ & 1.00 & $\begin{array}{l}0.93 \\
(0.42- \\
2.07), P= \\
0.86\end{array}$ & $\begin{array}{l}1.58 \\
(0.74- \\
3.40), P= \\
0.24\end{array}$ & $\begin{array}{l}2.01 \\
(1.01- \\
3.99) \\
P= \\
0.04\end{array}$ \\
\hline $\begin{array}{l}\text { Order } \\
\text { cohort }^{\$}\end{array}$ & 251 & 504 & 655 & 891 & 868 & 661 & 1014 \\
\hline $\begin{array}{l}\text { No. of } \\
\text { deaths }\end{array}$ & $2(0.008)$ & $9(0.018)$ & $6(0.009)$ & $\begin{array}{l}7 \\
(0.008)\end{array}$ & $8(0.009)$ & $12(0.018)$ & $\begin{array}{l}26 \\
(0.026)\end{array}$ \\
\hline $\begin{array}{l}\text { Model } 1 \\
(\mathrm{HR}, \\
95 \% \mathrm{Cl})\end{array}$ & $\begin{array}{l}1.02(0.21- \\
4.89), P= \\
0.98\end{array}$ & $\begin{array}{l}2.21 \\
(0.82- \\
5.95), P= \\
0.12\end{array}$ & $\begin{array}{l}1.15 \\
(0.39- \\
3.43), P= \\
0.80\end{array}$ & 1.00 & $\begin{array}{l}1.15 \\
(0.42- \\
3.16), P= \\
0.79\end{array}$ & $\begin{array}{l}2.29 \\
(0.90- \\
5.80, P= \\
0.08\end{array}$ & $\begin{array}{l}3.40 \\
(1.48- \\
7.84), \\
P= \\
0.004\end{array}$ \\
\hline $\begin{array}{l}\text { Model } 2 \\
(\mathrm{HR}, \\
95 \% \mathrm{Cl})\end{array}$ & $\begin{array}{l}1.19(0.24- \\
5.81), P= \\
0.83\end{array}$ & $\begin{array}{l}2.40 \\
(0.88- \\
6.51), P= \\
0.09\end{array}$ & $\begin{array}{l}1.21 \\
(0.40- \\
3.64), P= \\
0.73\end{array}$ & 1.00 & $\begin{array}{l}1.03 \\
(0.37- \\
2.86), P= \\
0.95\end{array}$ & $\begin{array}{l}2.01 \\
(0.78- \\
5.14), P= \\
0.15\end{array}$ & $\begin{array}{l}2.93 \\
(1.26- \\
6.82), \\
P= \\
0.01\end{array}$ \\
\hline \multicolumn{8}{|c|}{$\begin{array}{l}\text { \$: Postmenopausal women born before } 1940 \text { were analysed; } \& \text { :Postmenopausal women born in } 1940 \\
\text { or later were analysed; model 1: a crude hazard ratio model without adjustments; model } 2: \text { a } \\
\text { multivariate adjusted model including age, diabetes, hypertension, dyslipidaemia, smoking, alcohol } \\
\text { drinking, physical activity, body mass index, self-rated health, education, job, family income, number } \\
\text { of children and oral contraceptive pill use. }\end{array}$} \\
\hline
\end{tabular}




\begin{tabular}{|c|c|c|c|c|c|c|c|}
\hline & \multicolumn{7}{|c|}{ Age at menarche (years) } \\
\hline & $\leq 12$ & 13 & 14 & 15 & 16 & 17 & $\geq 18$ \\
\hline $\begin{array}{l}\text { Younger } \\
\text { cohort \& }^{\&}\end{array}$ & 1106 & 2147 & 2174 & 1883 & 1772 & 1037 & 1414 \\
\hline $\begin{array}{l}\text { No. of } \\
\text { deaths }\end{array}$ & $1(0.001)$ & $8(0.004)$ & $2(0.001)$ & $\begin{array}{l}5 \\
(0.003)\end{array}$ & $4(0.002)$ & $3(0.003)$ & $\begin{array}{l}2 \\
(0.001)\end{array}$ \\
\hline $\begin{array}{l}\text { Model } 1 \\
(\mathrm{HR}, \\
95 \% \mathrm{Cl})\end{array}$ & $\begin{array}{l}0.35(0.04- \\
3.03), P= \\
0.34\end{array}$ & $\begin{array}{l}1.42 \\
(0.46- \\
4.33), P= \\
0.54\end{array}$ & $\begin{array}{l}0.35 \\
(0.07- \\
1.82), P= \\
0.21\end{array}$ & 1.00 & $\begin{array}{l}0.86 \\
(0.23- \\
3.19), P= \\
0.82\end{array}$ & $\begin{array}{l}1.09 \\
(0.26- \\
4.56), P= \\
0.91\end{array}$ & $\begin{array}{l}0.53 \\
(0.10- \\
2.73) \\
P= \\
0.45\end{array}$ \\
\hline $\begin{array}{l}\text { Model } 2 \\
(\mathrm{HR}, \\
95 \% \mathrm{Cl})\end{array}$ & $\begin{array}{l}0.38(0.04- \\
3.33), P= \\
0.39\end{array}$ & $\begin{array}{l}1.61 \\
(0.52- \\
5.02), P= \\
0.41\end{array}$ & $\begin{array}{l}0.36 \\
(0.07- \\
1.86), P= \\
0.22\end{array}$ & 1.00 & $\begin{array}{l}0.82 \\
(0.22- \\
3.07), P= \\
0.75\end{array}$ & $\begin{array}{l}1.01 \\
(0.24- \\
4.29), P= \\
0.98\end{array}$ & $\begin{array}{l}0.49 \\
(0.09- \\
2.57) \\
P= \\
0.40\end{array}$ \\
\hline \multicolumn{8}{|c|}{ Haemorrhagic stroke } \\
\hline Cohort & 1361 & 2638 & 2834 & 2770 & 2648 & 1700 & 2413 \\
\hline $\begin{array}{l}\text { No. of } \\
\text { deaths }\end{array}$ & $7(0.005)$ & $4(0.002)$ & $\begin{array}{l}13 \\
(0.005)\end{array}$ & $\begin{array}{l}8 \\
(0.003)\end{array}$ & $20(0.008)$ & $17(0.01)$ & $\begin{array}{l}13 \\
(0.005)\end{array}$ \\
\hline $\begin{array}{l}\text { Model } 1 \\
(\mathrm{HR}, \\
95 \% \mathrm{Cl})\end{array}$ & $\begin{array}{l}1.92(0.70- \\
5.29, P= \\
0.21\end{array}$ & $\begin{array}{l}0.54(0.16- \\
1.78), P= \\
0.31\end{array}$ & $\begin{array}{l}1.64 \\
(0.68- \\
3.96), P= \\
0.27\end{array}$ & 1.00 & $\begin{array}{l}2.61 \\
(1.15- \\
5.93), P= \\
0.02\end{array}$ & $\begin{array}{l}3.44 \\
(1.49- \\
7.98), P= \\
0.004\end{array}$ & $\begin{array}{l}1.87 \\
(0.77- \\
4.50) \\
P= \\
0.17\end{array}$ \\
\hline $\begin{array}{l}\text { Model } 2 \\
(\mathrm{HR}, \\
95 \% \mathrm{Cl})\end{array}$ & $\begin{array}{l}2.75(0.99- \\
7.69), P= \\
0.05\end{array}$ & $\begin{array}{l}0.72 \\
(0.22- \\
2.40), P= \\
0.59\end{array}$ & $\begin{array}{l}2.01 \\
(0.83- \\
4.88), P= \\
0.12\end{array}$ & 1.00 & $\begin{array}{l}2.24 \\
(0.98- \\
5.10), P= \\
0.06\end{array}$ & $\begin{array}{l}2.65 \\
(1.14- \\
6.18), P= \\
0.02\end{array}$ & $\begin{array}{l}1.32 \\
(0.54- \\
3.21) \\
P= \\
0.54\end{array}$ \\
\hline $\begin{array}{l}\text { Order } \\
\text { cohort\$ }\end{array}$ & 255 & 497 & 658 & 888 & 875 & 660 & 1000 \\
\hline $\begin{array}{l}\text { No. of } \\
\text { deaths }\end{array}$ & $6(0.024)$ & $2(0.004)$ & $9(0.014)$ & $\begin{array}{l}4 \\
(0.004)\end{array}$ & $15(0.017)$ & $11(0.017)$ & $\begin{array}{l}12 \\
(0.012)\end{array}$ \\
\hline $\begin{array}{l}\text { Model } 1 \\
(\mathrm{HR}, \\
95 \% \mathrm{Cl})\end{array}$ & $\begin{array}{l}5.31(1.50- \\
18.83), P= \\
0.01\end{array}$ & $\begin{array}{l}0.87 \\
(0.16- \\
4.77), P= \\
0.88\end{array}$ & $\begin{array}{l}3.01 \\
(0.93- \\
9.78), P= \\
0.07\end{array}$ & 1.00 & $\begin{array}{l}3.74(1.24- \\
11.28), P= \\
0.02\end{array}$ & $\begin{array}{l}3.65(1.16- \\
11.46), P= \\
0.03\end{array}$ & $\begin{array}{l}2.73 \\
(0.88- \\
8.45) \\
P= \\
0.08\end{array}$ \\
\hline \multicolumn{8}{|c|}{$\begin{array}{l}\text { \$: Postmenopausal women born before } 1940 \text { were analysed; \&:Postmenopausal women born in } 1940 \\
\text { or later were analysed; model 1: a crude hazard ratio model without adjustments; model 2: a } \\
\text { multivariate adjusted model including age, diabetes, hypertension, dyslipidaemia, smoking, alcohol } \\
\text { drinking, physical activity, body mass index, self-rated health, education, job, family income, number } \\
\text { of children and oral contraceptive pill use. }\end{array}$} \\
\hline
\end{tabular}




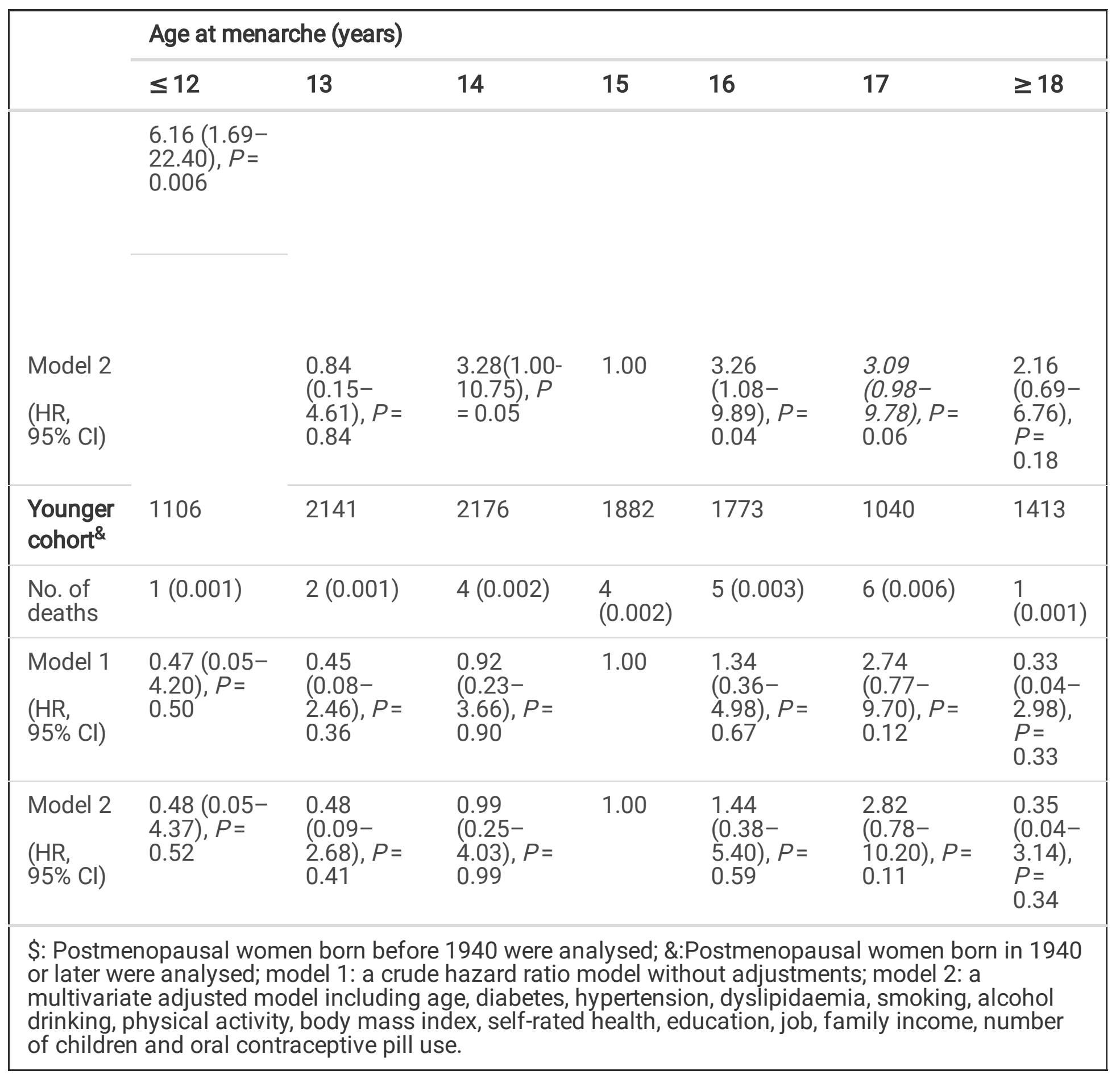


Table 3

Association between fatal stroke occurrence and age at menarche after a series of exclusions in the GBCS

\begin{tabular}{|c|c|c|c|c|c|c|c|}
\hline & \multicolumn{7}{|c|}{ Age at menarche (years) } \\
\hline & $\leq 12$ & 13 & 14 & 15 & 16 & 17 & $\geq 18$ \\
\hline \multicolumn{8}{|l|}{ Stroke } \\
\hline Cohort & 1099 & 2209 & 2388 & 2394 & 2322 & 1498 & 2175 \\
\hline $\begin{array}{l}\text { No. of } \\
\text { deaths }\end{array}$ & $10(0.010)$ & $25(0.021)$ & $\begin{array}{l}26 \\
(0.011)\end{array}$ & $22(0.009)$ & $\begin{array}{l}34 \\
(0.015)\end{array}$ & $\begin{array}{l}30 \\
(0.02)\end{array}$ & $\begin{array}{l}46 \\
(0.021)\end{array}$ \\
\hline $\begin{array}{l}\text { Model } \\
1 \\
(\mathrm{HR}, \\
95 \% \\
\mathrm{Cl})\end{array}$ & $\begin{array}{l}1.07(0.51- \\
2.25), P= \\
0.87\end{array}$ & $\begin{array}{l}1.26 \\
(0.71- \\
2.23), P= \\
0.44\end{array}$ & $\begin{array}{l}1.23(0.70- \\
2.16), P= \\
0.48\end{array}$ & 1.00 & $\begin{array}{l}1.59 \\
(0.93- \\
2.72), P \\
=0.09\end{array}$ & $\begin{array}{l}2.18 \\
(1.26- \\
3.79), P \\
=0.005\end{array}$ & $\begin{array}{l}2.32 \\
(1.40- \\
3.86), P \\
=0.001\end{array}$ \\
\hline $\begin{array}{l}\text { Model } \\
2 \\
(\mathrm{HR}, \\
95 \% \\
\mathrm{Cl})\end{array}$ & $\begin{array}{l}1.56(0.73- \\
3.31), P= \\
0.25\end{array}$ & $\begin{array}{l}1.75 \\
(0.98- \\
3.13), P= \\
0.06\end{array}$ & $\begin{array}{l}1.51(0.86- \\
2.68), P= \\
0.16\end{array}$ & 1.00 & $\begin{array}{l}1.38 \\
(0.80- \\
2.36), P \\
=0.25\end{array}$ & $\begin{array}{l}1.70 \\
(0.97- \\
2.95), P \\
=0.06\end{array}$ & $\begin{array}{l}1.69 \\
(1.01- \\
2.82), P \\
=0.04\end{array}$ \\
\hline $\begin{array}{l}\text { Order } \\
\text { cohorts }\end{array}$ & 227 & 453 & 591 & 807 & 814 & 614 & 944 \\
\hline $\begin{array}{l}\text { No. of } \\
\text { deaths }\end{array}$ & $10(0.044)$ & $14(0.031)$ & $\begin{array}{l}19 \\
(0.032)\end{array}$ & $\begin{array}{l}12 \\
(0.015)\end{array}$ & $22(0.027)$ & $\begin{array}{l}24 \\
(0.039)\end{array}$ & $\begin{array}{l}42 \\
(0.044)\end{array}$ \\
\hline $\begin{array}{l}\text { Model } \\
1 \\
(\mathrm{HR}, \\
95 \% \\
\mathrm{Cl})\end{array}$ & $\begin{array}{l}3.01 \\
(1.30- \\
6.98), P= \\
0.01\end{array}$ & $\begin{array}{l}2.06 \\
(0.95- \\
4.45), P= \\
0.07\end{array}$ & $\begin{array}{l}2.17(1.05- \\
4.47), P= \\
0.04\end{array}$ & 1.00 & $\begin{array}{l}1.77 \\
(0.88- \\
3.58), P \\
=0.11\end{array}$ & $\begin{array}{l}2.61 \\
(1.31- \\
5.23), P \\
=0.007\end{array}$ & $\begin{array}{l}3.11 \\
(1.64- \\
5.91), P \\
=0.001\end{array}$ \\
\hline $\begin{array}{l}\text { Model } \\
2 \\
(\mathrm{HR}, \\
95 \% \\
\mathrm{Cl})\end{array}$ & $\begin{array}{l}3.67 \\
(1.56- \\
8.60), P= \\
0.003\end{array}$ & $\begin{array}{l}2.23 \\
(1.02- \\
4.85), P= \\
0.04\end{array}$ & $\begin{array}{l}2.37(1.14- \\
4.88), P= \\
0.02\end{array}$ & 1.00 & $\begin{array}{l}1.57 \\
(0.77- \\
3.18), P \\
=0.21\end{array}$ & $\begin{array}{l}2.30 \\
(1.14- \\
4.62), P \\
=0.02\end{array}$ & $\begin{array}{l}2.56 \\
(1.34- \\
4.89), P \\
=0.004\end{array}$ \\
\hline \multicolumn{8}{|c|}{ Ischaemic stroke } \\
\hline Cohort & 1090 & 2200 & 2369 & 2383 & 2299 & 1482 & 2155 \\
\hline
\end{tabular}

\$: Postmenopausal women born before 1940 were analyzed; model 1: a crude hazard ratio model without adjustments; model 2: a multivariate adjust model including age, diabetes, hypertension, dyslipidemia, smoking, alcohol drinking, physical activity, body mass index, self-rated health, education, job, family income, number of children and oral contraceptive pill use. 


\begin{tabular}{|c|c|c|c|c|c|c|c|}
\hline & \multicolumn{7}{|c|}{ Age at menarche (years) } \\
\hline & $\leq 12$ & 13 & 14 & 15 & 16 & 17 & $\geq 18$ \\
\hline $\begin{array}{l}\text { No. of } \\
\text { deaths }\end{array}$ & $1(0.001)$ & $16(0.007)$ & $7(0.003)$ & $11(0.005)$ & $\begin{array}{l}11 \\
(0.005)\end{array}$ & $\begin{array}{l}14 \\
(0.009)\end{array}$ & $\begin{array}{l}26 \\
(0.012)\end{array}$ \\
\hline $\begin{array}{l}\text { Model } \\
1 \\
(\mathrm{HR}, \\
95 \% \\
\mathrm{Cl})\end{array}$ & $\begin{array}{l}0.21 \\
(0.03- \\
1.65), P= \\
0.14\end{array}$ & $\begin{array}{l}1.61 \\
(0.75- \\
3.46), P= \\
0.23\end{array}$ & $\begin{array}{l}0.66(0.26- \\
1.71), P= \\
0.39\end{array}$ & 1.00 & $\begin{array}{l}1.03 \\
(0.45- \\
2.38), P \\
=0.94\end{array}$ & $\begin{array}{l}2.05 \\
(0.93- \\
4.51), P \\
=0.08\end{array}$ & $\begin{array}{l}2.64 \\
(1.30- \\
5.33), P \\
=0.007\end{array}$ \\
\hline $\begin{array}{l}\text { Model } \\
2 \\
(\mathrm{HR}, \\
95 \% \\
\mathrm{Cl})\end{array}$ & $\begin{array}{l}0.30 \\
(0.04- \\
2.36), P= \\
0.25\end{array}$ & $\begin{array}{l}2.29 \\
(1.05- \\
4.99), P= \\
0.04\end{array}$ & $\begin{array}{l}0.78(0.30- \\
2.04), P= \\
0.62\end{array}$ & 1.00 & $\begin{array}{l}0.91 \\
(0.39- \\
2.11), P \\
=0.83\end{array}$ & $\begin{array}{l}1.60 \\
(0.72- \\
3.55), P \\
=0.25\end{array}$ & $\begin{array}{l}1.98 \\
(0.97- \\
4.03), P \\
=0.06\end{array}$ \\
\hline $\begin{array}{l}\text { Order } \\
\text { cohort }\end{array}$ & 218 & 448 & 578 & 802 & 799 & 601 & 926 \\
\hline $\begin{array}{l}\text { No. of } \\
\text { deaths }\end{array}$ & $1(0.005)$ & $9(0.02)$ & $6(0.01)$ & $7(0.006)$ & $\begin{array}{l}7 \\
(0.009)\end{array}$ & $\begin{array}{l}11 \\
(0.018)\end{array}$ & $\begin{array}{l}24 \\
(0.025)\end{array}$ \\
\hline $\begin{array}{l}\text { Model } \\
1 \\
(\mathrm{HR}, \\
95 \% \\
\mathrm{Cl})\end{array}$ & $\begin{array}{l}0.53 \\
(0.07- \\
4.27), P= \\
0.55\end{array}$ & $\begin{array}{l}2.28 \\
(0.85- \\
6.13), P= \\
0.10\end{array}$ & $\begin{array}{l}1.19(0.40- \\
3.54), P= \\
0.76\end{array}$ & 1.00 & $\begin{array}{l}0.97 \\
(0.34- \\
2.78), P \\
=0.96\end{array}$ & $\begin{array}{l}2.08 \\
(0.81- \\
5.37), P \\
=0.13\end{array}$ & $\begin{array}{l}3.08 \\
(1.33- \\
7.16, P= \\
0.009\end{array}$ \\
\hline $\begin{array}{l}\text { Model } \\
2 \\
(\mathrm{HR}, \\
95 \% \\
\mathrm{Cl})\end{array}$ & $\begin{array}{l}0.63 \\
(0.08- \\
5.21), P= \\
0.67\end{array}$ & $\begin{array}{l}2.56 \\
(0.94- \\
6.94), P= \\
0.07\end{array}$ & $\begin{array}{l}1.25(0.42- \\
3.74), P= \\
0.70\end{array}$ & 1.00 & $\begin{array}{l}0.89 \\
(0.31- \\
2.56), P \\
=0.83\end{array}$ & $\begin{array}{l}1.81 \\
(0.70- \\
4.73), P \\
=0.22\end{array}$ & $\begin{array}{l}2.61 \\
(1.11- \\
6.12), P \\
=0.03\end{array}$ \\
\hline \multicolumn{8}{|c|}{ Haemorrhagic stroke } \\
\hline Cohort & 1095 & 2187 & 2374 & 2380 & 2305 & 1481 & 2140 \\
\hline $\begin{array}{l}\text { No. of } \\
\text { deaths }\end{array}$ & $6(0.0055)$ & $3(0.0014)$ & $\begin{array}{l}12 \\
(0.005)\end{array}$ & $8(0.003)$ & $\begin{array}{l}17 \\
(0.007)\end{array}$ & $\begin{array}{l}13 \\
(0.009)\end{array}$ & $\begin{array}{l}11 \\
(0.005)\end{array}$ \\
\hline
\end{tabular}

\$: Postmenopausal women born before 1940 were analyzed; model 1: a crude hazard ratio model without adjustments; model 2: a multivariate adjust model including age, diabetes, hypertension, dyslipidemia, smoking, alcohol drinking, physical activity, body mass index, self-rated health, education, job, family income, number of children and oral contraceptive pill use. 


\begin{tabular}{|c|c|c|c|c|c|c|c|}
\hline & \multicolumn{7}{|c|}{ Age at menarche (years) } \\
\hline & $\leq 12$ & 13 & 14 & 15 & 16 & 17 & $\geq 18$ \\
\hline $\begin{array}{l}\text { Model } \\
1 \\
(\mathrm{HR}, \\
95 \% \\
\mathrm{Cl})\end{array}$ & $\begin{array}{l}1.77 \\
(0.61- \\
5.10), P= \\
0.29\end{array}$ & $\begin{array}{l}0.42 \\
(0.11- \\
1.57), P= \\
0.20\end{array}$ & $\begin{array}{l}1.56(0.64- \\
3.82), P= \\
0.33\end{array}$ & 1.00 & $\begin{array}{l}2.19 \\
(0.94- \\
5.07), P \\
=0.07\end{array}$ & $\begin{array}{l}2.61 \\
(1.08- \\
6.30), P \\
=0.03\end{array}$ & $\begin{array}{l}1.53 \\
(0.62- \\
3.81), P \\
=0.36\end{array}$ \\
\hline $\begin{array}{l}\text { Model } \\
2 \\
(\mathrm{HR}, \\
95 \% \\
\mathrm{Cl})\end{array}$ & $\begin{array}{l}2.38 \\
(0.81- \\
6.98), P= \\
0.11\end{array}$ & $\begin{array}{l}0.55 \\
(0.15- \\
2.09), P= \\
0.38\end{array}$ & $\begin{array}{l}1.90(0.77- \\
4.69), P= \\
0.16\end{array}$ & 1.00 & $\begin{array}{l}1.91 \\
(0.82- \\
4.44), P \\
=0.13\end{array}$ & $\begin{array}{l}2.09 \\
(0.86- \\
5.07), P \\
=0.11\end{array}$ & $\begin{array}{l}1.13 \\
(0.45- \\
2.84), P \\
=0.79\end{array}$ \\
\hline $\begin{array}{l}\text { Order } \\
\text { cohort } \$\end{array}$ & 223 & 440 & 580 & 799 & 804 & 600 & 912 \\
\hline $\begin{array}{l}\text { No. of } \\
\text { deaths }\end{array}$ & $6(0.027)$ & $1(0.002)$ & $8(0.014)$ & $4(0.005)$ & $\begin{array}{l}12 \\
(0.015)\end{array}$ & $\begin{array}{l}10 \\
(0.017)\end{array}$ & $\begin{array}{l}10 \\
(0.011)\end{array}$ \\
\hline $\begin{array}{l}\text { Model } \\
1 \\
(\mathrm{HR}, \\
95 \% \\
\mathrm{Cl})\end{array}$ & $\begin{array}{l}5.45(1.54- \\
19.32), P= \\
0.009\end{array}$ & $\begin{array}{l}0.45 \\
(0.05- \\
4.01), P= \\
0.47\end{array}$ & $\begin{array}{l}2.76(0.83- \\
9.16), P= \\
0.10\end{array}$ & 1.00 & $\begin{array}{l}2.91 \\
(0.94- \\
9.02), P \\
=0.07\end{array}$ & $\begin{array}{l}3.29 \\
(1.03- \\
10.48) \\
P=0.04\end{array}$ & $\begin{array}{l}2.23 \\
(0.70- \\
7.12), P \\
=0.17\end{array}$ \\
\hline $\begin{array}{l}\text { Model } \\
2 \\
(\mathrm{HR}, \\
95 \% \\
\mathrm{Cl})\end{array}$ & $\begin{array}{l}6.22(1.70- \\
22.76), P= \\
0.006\end{array}$ & $\begin{array}{l}0.45 \\
(0.05- \\
4.08), P= \\
0.48\end{array}$ & $\begin{array}{l}3.03(0.90- \\
10.20), P= \\
0.07\end{array}$ & 1.00 & $\begin{array}{l}2.62 \\
(0.84- \\
8.18), P \\
=0.10\end{array}$ & $\begin{array}{l}2.93 \\
(0.91- \\
9.48), P \\
=0.07\end{array}$ & $\begin{array}{l}1.85 \\
(0.57- \\
5.96), P \\
=0.30\end{array}$ \\
\hline $\begin{array}{l}\text { \$: Postr } \\
\text { without } \\
\text { dyslipid } \\
\text { educati }\end{array}$ & $\begin{array}{l}\text { opausal w } \\
\text { ustments; } \\
\text { a, smoking } \\
\text { ob, family }\end{array}$ & $\begin{array}{l}\text { born be } \\
\text { el 2: a m } \\
\text { ohol drin } \\
\text { me, num }\end{array}$ & $\begin{array}{l}1940 \text { wer } \\
\text { riate adju } \\
\text { physical } \\
\text { f children }\end{array}$ & $\begin{array}{l}\text { lyzed; } n \\
\text { odel inc } \\
\text { ity, body } \\
\text { oral con }\end{array}$ & $\begin{array}{l}\text { : a cruc } \\
\text { age, dia } \\
\text { index, } \\
\text { tive pill }\end{array}$ & $\begin{array}{l}\text { azard rat } \\
\text { es, hyper } \\
\text { rated he } \\
\text {. }\end{array}$ & $\begin{array}{l}\text { nodel } \\
\text { sion, }\end{array}$ \\
\hline
\end{tabular}

\section{Menopause In Relation To Fatal Stroke Occurrence}

Table 4 shows menopause in relation to fatal stroke occurrence. Compared with menopausal women aged 51-52 years, postmenopausal women born before 1940 showed a significant association with the risk of fatal stroke $(\mathrm{HR}=1.97,95 \% \mathrm{Cl} 1.05-3.69, \mathrm{P}=0.03)$ only. The association was still observed in a sensitivity analysis after excluding uterus removal, ovaries, breast masses (benign or malignant) or hormone replacement therapy (Table 5). 
Table 4

Association between fatal stroke occurrence and age at menopause in the GBCS

\begin{tabular}{|c|c|c|c|c|c|}
\hline & \multicolumn{5}{|c|}{ Age at menopause (years) } \\
\hline & $<43$ & $43-47$ & $48-50$ & $51-52$ & $\geq 53$ \\
\hline Cohort & 940 & 2805 & 6600 & 2928 & 3231 \\
\hline $\begin{array}{l}\text { No. of } \\
\text { deaths }\end{array}$ & $26(0.028)$ & $42(0.015)$ & $88(0.013)$ & $\begin{array}{l}31 \\
(0.010)\end{array}$ & $35(0.010)$ \\
\hline $\begin{array}{l}\text { Model } 1 \\
(\mathrm{HR}, \\
95 \% \mathrm{Cl})\end{array}$ & $\begin{array}{l}2.58(1.53-4.35) \\
P<0.001\end{array}$ & $\begin{array}{l}1.40(0.88-2.23) \\
P=0.16\end{array}$ & $\begin{array}{l}1.25(0.83-1.88) \\
P=0.29\end{array}$ & 1.00 & $\begin{array}{l}0.98(0.61- \\
1.59), P=0.94\end{array}$ \\
\hline $\begin{array}{l}\text { Model } 2 \\
(\mathrm{HR}, \\
95 \% \mathrm{Cl})\end{array}$ & $\begin{array}{l}1.64(0.97-2.78) \\
P=0.06\end{array}$ & $\begin{array}{l}1.09(0.69-1.74) \\
P=0.71\end{array}$ & $\begin{array}{l}1.10(0.73-1.65), \\
P=0.66\end{array}$ & 1.00 & $\begin{array}{l}0.99(0.61- \\
1.61), P=0.96\end{array}$ \\
\hline $\begin{array}{l}\text { Order } \\
\text { cohort } \$\end{array}$ & 413 & 981 & 1996 & 752 & 796 \\
\hline $\begin{array}{l}\text { No. of } \\
\text { deaths }\end{array}$ & $22(0.053)$ & $36(0.037)$ & $62(0.031)$ & $\begin{array}{l}18 \\
(0.024)\end{array}$ & $26(0.033)$ \\
\hline $\begin{array}{l}\text { Model } 1 \\
(\mathrm{HR}, \\
95 \% \mathrm{Cl})\end{array}$ & $\begin{array}{l}2.30(1.23-4.28) \\
P=0.009\end{array}$ & $\begin{array}{l}1.52(0.86-2.67) \\
P=0.15\end{array}$ & $\begin{array}{l}1.29(0.77-2.18) \\
P=0.34\end{array}$ & 1.00 & $\begin{array}{l}1.33(0.73- \\
2.42), P=0.35\end{array}$ \\
\hline $\begin{array}{l}\text { Model } 2 \\
(\mathrm{HR}, \\
95 \% \mathrm{Cl})\end{array}$ & $\begin{array}{l}1.97(1.05-3.69) \\
P=0.03\end{array}$ & $\begin{array}{l}1.43(0.81-2.52) \\
P=0.22\end{array}$ & $\begin{array}{l}1.25(0.74-2.11) \\
P=0.41\end{array}$ & 1.00 & $\begin{array}{l}1.35(0.74- \\
2.47), P=0.33\end{array}$ \\
\hline $\begin{array}{l}\text { \$: Postm } \\
\text { without a } \\
\text { dyslipida } \\
\text { educatio }\end{array}$ & $\begin{array}{l}\text { pausal women b } \\
\text { sttments; model } 2 \\
\text { ia, smoking, alcol } \\
\text { b, family income }\end{array}$ & $\begin{array}{l}\text { pefore } 1940 \text { were } \\
\text { nultivariate adjus } \\
\text { Irinking, physical } \\
\text { nber of children }\end{array}$ & $\begin{array}{l}\text { lalysed; model } 1 \text { : } \\
\text { model including } \\
\text { tivity, body mass } \\
\text { oral contraceptiv }\end{array}$ & $\begin{array}{l}\text { ude haz } \\
\text { e, diabet } \\
\text { ex, self-rr } \\
\text { ill use. }\end{array}$ & $\begin{array}{l}\text { d ratio model } \\
\text { hypertension, } \\
\text { ed health, }\end{array}$ \\
\hline
\end{tabular}


Table 5

Association between fatal stroke occurrence and age at menopause after a series of exclusions in the GBCS

\begin{tabular}{|c|c|c|c|c|c|}
\hline & \multicolumn{5}{|c|}{ Age at menopause (years) } \\
\hline & $<43$ & $43-47$ & $48-50$ & $51-52$ & $\geq 53$ \\
\hline Cohort & 662 & 2201 & 5722 & 2609 & 2891 \\
\hline $\begin{array}{l}\text { No. of } \\
\text { deaths }\end{array}$ & $23(0.035)$ & $33(0.015)$ & $77(0.013)$ & $28(0.011)$ & $32(0.011)$ \\
\hline $\begin{array}{l}\text { Model } 1 \\
\text { (HR, 95\% } \\
\mathrm{Cl})\end{array}$ & $\begin{array}{l}3.17(1.83- \\
5.51), P<0.001\end{array}$ & $\begin{array}{l}1.38(0.83- \\
2.29), P=0.21\end{array}$ & $\begin{array}{l}1.24(0.80- \\
1.91), P=0.33\end{array}$ & $\begin{array}{l}0.99(0.60- \\
1.65), P=0.97\end{array}$ & \\
\hline $\begin{array}{l}\text { Model } 2 \\
\text { (HR, 95\% } \\
\mathrm{Cl})\end{array}$ & $\begin{array}{l}1.79(1.03- \\
3.14), P=0.04\end{array}$ & $\begin{array}{l}1.02(0.61- \\
1.69), P=0.95\end{array}$ & $\begin{array}{l}1.08(0.70- \\
1.67), P=0.73\end{array}$ & 1 & $\begin{array}{l}1.02(0.61- \\
1.69), P=0.95\end{array}$ \\
\hline $\begin{array}{l}\text { Order } \\
\text { cohort\$ }\end{array}$ & 348 & 864 & 1816 & 698 & 724 \\
\hline $\begin{array}{l}\text { No. of } \\
\text { deaths }\end{array}$ & $19(0.055)$ & $29(0.034)$ & $55(0.030)$ & $16(0.023)$ & $24(0.033)$ \\
\hline $\begin{array}{l}\text { Model } 1 \\
\text { (HR, 95\% } \\
\mathrm{Cl})\end{array}$ & $\begin{array}{l}2.49(1.28- \\
4.84), P=0.007\end{array}$ & $\begin{array}{l}1.45(0.79- \\
2.67), P=0.23\end{array}$ & $\begin{array}{l}1.31(0.75- \\
2.29), P=0.34\end{array}$ & 1 & $\begin{array}{l}1.40(0.75- \\
2.64), P=0.29\end{array}$ \\
\hline $\begin{array}{l}\text { Model } 2 \\
\text { (HR, 95\% } \\
\text { Cl) }\end{array}$ & $\begin{array}{l}2.07(1.06- \\
4.06), P=0.03\end{array}$ & $\begin{array}{l}1.37(0.74- \\
2.54), P=0.31\end{array}$ & $\begin{array}{l}1.28(0.73- \\
2.24), P=0.39\end{array}$ & 1 & $\begin{array}{l}1.46(0.77- \\
2.75), P=0.24\end{array}$ \\
\hline
\end{tabular}

\$: Postmenopausal women born before 1940 were analyzed; model 1: a crude hazard ratio model without adjustments; model 2: a multivariate adjust model including age, diabetes, hypertension, dyslipidemia, smoking, alcohol drinking, physical activity, body mass index, self-rated health, education, job, family income, number of children and oral contraceptive pill use.

\section{Reproductive Years In Relation To Fatal Stroke Occurrence}

Table 6 shows reproductive years in relation to fatal stroke occurrence. Compared with those with a number of reproductive years from 32-34, those with a number of reproductive years $\leq 28$ had an increased risk of fatal stroke $(H R=1.91,95 \% \mathrm{Cl} 1.28-2.86, \mathrm{P}=0.001)$ and ischaemic stroke $(H R=2.26$, $95 \% \mathrm{Cl} 1.26-4.05, \mathrm{P}=0.006)$ in the whole cohort, and similar associations were found for fatal stroke $(H R=1.79,95 \% \mathrm{Cl} 1.15-2.80, \mathrm{P}=0.01)$ and fatal ischaemic stroke $(\mathrm{HR}=2.21,95 \% \mathrm{Cl} 1.18-4.16, \mathrm{P}=0.01)$ in the cohort of postmenopausal women born before 1940. Moreover, no reproductive years in relation to fatal stroke and fatal ischaemic stroke were observed in the cohort born in 1940 or later. Such associations were still observed in a sensitivity analysis after exclusion of those who had removal of the 
uterus, ovaries, or breast masses (benign or malignant) or received hormone replacement therapy (Table 7). 
Table 6

Association between fatal stroke occurrence and reproductive years in the GBCS

\section{Duration of reproductive years}

$\begin{array}{lllll}\leq 28 & 29-31 & 32-34 & 35-37 & \geq 38\end{array}$

Stroke

\begin{tabular}{|c|c|c|c|c|c|}
\hline Cohort & 1511 & 2193 & 4025 & 5114 & 3661 \\
\hline $\begin{array}{l}\text { No. of } \\
\text { deaths }\end{array}$ & $49(0.032)$ & $32(0.015)$ & $\begin{array}{l}49 \\
(0.012)\end{array}$ & $58(0.011)$ & $36(0.010)$ \\
\hline $\begin{array}{l}\text { Model } 1(\mathrm{HR}, \\
95 \% \mathrm{Cl})\end{array}$ & $\begin{array}{l}2.69(1.81-4.00) \\
P<0.001\end{array}$ & $\begin{array}{l}1.18(0.76- \\
1.85), P=0.46\end{array}$ & 1.00 & $\begin{array}{l}0.90(0.61- \\
1.33), P=0.60\end{array}$ & $\begin{array}{l}0.81(0.52- \\
1.24), P=0.33\end{array}$ \\
\hline $\begin{array}{l}\text { Model } 2(\mathrm{HR}, \\
95 \% \mathrm{Cl})\end{array}$ & $\begin{array}{l}1.91(1.28- \\
2.86), P=0.001\end{array}$ & $\begin{array}{l}0.97(0.62- \\
1.52), P=0.90\end{array}$ & 1.00 & $\begin{array}{l}1.09(0.74- \\
1.61), P=0.65\end{array}$ & $\begin{array}{l}1.11(0.72- \\
1.72), P=0.64\end{array}$ \\
\hline $\begin{array}{l}\text { Order } \\
\text { cohort\$ }\end{array}$ & 713 & 878 & 1283 & 1293 & 771 \\
\hline $\begin{array}{l}\text { No. of } \\
\text { deaths }\end{array}$ & $41(0.058)$ & $29(0.033)$ & $\begin{array}{l}38 \\
(0.030)\end{array}$ & $33(0.026)$ & $23(0.030)$ \\
\hline $\begin{array}{l}\text { Model } 1(\mathrm{HR}, \\
95 \% \mathrm{Cl})\end{array}$ & $\begin{array}{l}2.03(1.30- \\
3.15), P=0.002\end{array}$ & $\begin{array}{l}1.13(0.70- \\
1.83), P=0.62\end{array}$ & 1.00 & $\begin{array}{l}0.86(0.54- \\
1.36), P=0.51\end{array}$ & $\begin{array}{l}1.00(0.60- \\
1.68), P=0.99\end{array}$ \\
\hline $\begin{array}{l}\text { Model } 2(\mathrm{HR}, \\
95 \% \mathrm{Cl})\end{array}$ & $\begin{array}{l}1.79(1.15- \\
2.80), P=0.01\end{array}$ & $\begin{array}{l}1.05(0.65- \\
1.71), P=0.83\end{array}$ & 1.00 & $\begin{array}{l}0.89(0.56- \\
1.42), P=0.62\end{array}$ & $\begin{array}{l}1.14(0.67- \\
1.93), P=0.62\end{array}$ \\
\hline $\begin{array}{l}\text { Younger } \\
\text { cohort }^{\&}\end{array}$ & 798 & 1315 & 2742 & 3821 & 2890 \\
\hline $\begin{array}{l}\text { No. of } \\
\text { deaths }\end{array}$ & $8(0.010)$ & $3(0.002)$ & $\begin{array}{l}11 \\
(0.004)\end{array}$ & $25(0.006)$ & $13(0.004)$ \\
\hline $\begin{array}{l}\text { Model } 1(\mathrm{HR}, \\
95 \% \mathrm{Cl})\end{array}$ & $\begin{array}{l}2.51(1.01- \\
6.25), P=0.047\end{array}$ & $\begin{array}{l}0.56(0.16- \\
2.01), P=0.37\end{array}$ & 1.00 & $\begin{array}{l}1.50(0.73- \\
3.07), P=0.27\end{array}$ & $\begin{array}{l}1.10(0.50- \\
2.47), P=0.81\end{array}$ \\
\hline $\begin{array}{l}\text { Model } 2(\mathrm{HR}, \\
95 \% \mathrm{Cl})\end{array}$ & $\begin{array}{l}2.11(0.84- \\
5.27), P=0.11\end{array}$ & $\begin{array}{l}0.52(0.14- \\
1.86), P=0.31\end{array}$ & 1.00 & $\begin{array}{l}1.63(0.79- \\
3.36), P=0.19\end{array}$ & $\begin{array}{l}1.13(0.51- \\
2.55), P=0.76\end{array}$ \\
\hline \multicolumn{6}{|c|}{ Ischaemic stroke } \\
\hline Cohort & 1486 & 2173 & 3998 & 5082 & 3638 \\
\hline $\begin{array}{l}\text { No. of } \\
\text { deaths }\end{array}$ & $24(0.016)$ & $12(0.006)$ & $\begin{array}{l}22 \\
(0.006)\end{array}$ & $24(0.005)$ & $13(0.004)$ \\
\hline
\end{tabular}

\$: Postmenopausal women born before 1940 were analyzed; \&:Postmenopausal women born in 1940 or later were analyzed; model 1: a crude hazard ratio model without adjustments; model 2: a multivariate adjust model including age, diabetes, hypertension, dyslipidemia, smoking, alcohol drinking, physical activity, body mass index, self-rated health, education, job, family income, number of children and oral contraceptive pill use. 


\begin{tabular}{|c|c|c|c|c|c|}
\hline & \multicolumn{5}{|c|}{ Duration of reproductive years } \\
\hline & $\leq 28$ & $29-31$ & $32-34$ & $35-37$ & $\geq 38$ \\
\hline $\begin{array}{l}\text { Model } 1(\mathrm{HR} \\
95 \% \mathrm{Cl})\end{array}$ & $\begin{array}{l}2.96(1.66-5.28) \\
P<0.001\end{array}$ & $\begin{array}{l}0.99(0.49- \\
2.00), P=0.98\end{array}$ & 1.00 & $\begin{array}{l}0.86(0.48- \\
1.54), P=0.61\end{array}$ & $\begin{array}{l}0.65(0.33- \\
1.29), P=0.22\end{array}$ \\
\hline $\begin{array}{l}\text { Model } 2(\mathrm{HR} \\
95 \% \mathrm{Cl})\end{array}$ & $\begin{array}{l}2.26(1.26- \\
4.05), P=0.006\end{array}$ & $\begin{array}{l}0.86(0.42- \\
1.73), P=0.67\end{array}$ & 1.00 & $\begin{array}{l}1.05(0.59- \\
1.88), P=0.87\end{array}$ & $\begin{array}{l}0.86(0.43- \\
1.83), P=0.68\end{array}$ \\
\hline $\begin{array}{l}\text { Older } \\
\text { cohort\$ }\end{array}$ & 694 & 859 & 1263 & 1272 & 756 \\
\hline $\begin{array}{l}\text { No. of } \\
\text { deaths }\end{array}$ & $22(0.032)$ & $10(0.012)$ & $\begin{array}{l}18 \\
(0.014)\end{array}$ & $12(0.009)$ & $8(0.011)$ \\
\hline $\begin{array}{l}\text { Model } 1 \text { (HR, } \\
95 \% \mathrm{Cl})\end{array}$ & $\begin{array}{l}2.32(1.25- \\
4.33), P=0.008\end{array}$ & $\begin{array}{l}0.83(0.38- \\
1.79), P=0.63\end{array}$ & 1.00 & $\begin{array}{l}0.66(0.32- \\
1.37), P=0.26\end{array}$ & $\begin{array}{l}0.73(0.32- \\
1.68), P=0.46\end{array}$ \\
\hline $\begin{array}{l}\text { Model } 2 \text { (HR, } \\
95 \% \mathrm{Cl})\end{array}$ & $\begin{array}{l}2.21(1.18- \\
4.16), P=0.01\end{array}$ & $\begin{array}{l}0.83(0.38- \\
1.79), P=0.63\end{array}$ & 1.00 & $\begin{array}{l}0.70(0.33- \\
1.45), P=0.33\end{array}$ & $\begin{array}{l}0.86(0.37- \\
1.99), P=0.72\end{array}$ \\
\hline $\begin{array}{l}\text { Younger } \\
\text { cohort }^{\&}\end{array}$ & 792 & 1314 & 2735 & 3810 & 2882 \\
\hline $\begin{array}{l}\text { No. of } \\
\text { deaths }\end{array}$ & $2(0.003)$ & $2(0.002)$ & $\begin{array}{l}4 \\
(0.0015)\end{array}$ & $12(0.003)$ & $5(0.002)$ \\
\hline $\begin{array}{l}\text { Model } 1 \text { (HR, } \\
95 \% \mathrm{Cl})\end{array}$ & $\begin{array}{l}1.74(0.32- \\
9.49), P=0.52\end{array}$ & $\begin{array}{l}1.03(0.19- \\
5.63), P=0.97\end{array}$ & 1.00 & $\begin{array}{l}2.16(0.70- \\
6.69), P=0.18\end{array}$ & $\begin{array}{l}1.18(0.32- \\
4.39), P=0.81\end{array}$ \\
\hline $\begin{array}{l}\text { Model } 2(\mathrm{HR} \\
95 \% \mathrm{Cl})\end{array}$ & $\begin{array}{l}1.51(0.28- \\
8.31), P=0.63\end{array}$ & $\begin{array}{l}0.95(0.17- \\
5.21), P=0.95\end{array}$ & 1.00 & $\begin{array}{l}2.33(0.75- \\
7.26), P=0.15\end{array}$ & $\begin{array}{l}1.20(0.32- \\
4.51), P=0.79\end{array}$ \\
\hline $\begin{array}{l}\text { \$: Postmenop } \\
\text { or later were ar } \\
\text { multivariate ac } \\
\text { drinking, physi } \\
\text { of children anc }\end{array}$ & $\begin{array}{l}\text { sal women born } \\
\text { yzed; model } 1: \\
\text { st model includ } \\
\text { I activity, body } r \\
\text { ral contraceptiv }\end{array}$ & $\begin{array}{l}\text { re } 1940 \text { were } \\
\text { de hazard rati } \\
\text { ge, diabetes, } \\
\text { index, self-rat } \\
\text { use. }\end{array}$ & $\begin{array}{l}\text { zed; \&: } \\
\text { lel with } \\
\text { ension, } \\
\text { alth, ed }\end{array}$ & $\begin{array}{l}\text { menopausal } \\
\text { adjustments; } \\
\text { lipidemia, smo } \\
\text { tion, job, famil }\end{array}$ & $\begin{array}{l}\text { en born in } 1940 \\
\text { lel 2: a } \\
\text { g, alcohol } \\
\text { come, number }\end{array}$ \\
\hline
\end{tabular}


Table 7

Association between stroke mortality and duration of reproductive years after a series of exclusions in the GBCS

\begin{tabular}{|c|c|c|c|c|c|}
\hline & \multicolumn{5}{|c|}{ Duration of reproductive years } \\
\hline & $\leq 28$ & $29-31$ & $32-34$ & $35-37$ & $\geq 38$ \\
\hline \multicolumn{6}{|l|}{ Stroke } \\
\hline Cohort & 1151 & 1815 & 3388 & 4482 & 3249 \\
\hline $\begin{array}{l}\text { No. of } \\
\text { deaths }\end{array}$ & $39(0.034)$ & $28(0.015)$ & $\begin{array}{l}42 \\
(0.012)\end{array}$ & $52(0.012)$ & $32(0.010)$ \\
\hline $\begin{array}{l}\text { Model } 1 \text { (HR, } \\
95 \% \mathrm{Cl})\end{array}$ & $\begin{array}{l}2.75(1.78- \\
4.26), P<0.001\end{array}$ & $\begin{array}{l}1.23(0.76- \\
1.98), P=0.40\end{array}$ & 1 & $\begin{array}{l}0.94(0.62- \\
1.41), P=0.76\end{array}$ & $\begin{array}{l}0.80(0.50- \\
1.26), P=0.33\end{array}$ \\
\hline $\begin{array}{l}\text { Model } 2(\mathrm{HR}, \\
95 \% \mathrm{Cl})\end{array}$ & $\begin{array}{l}1.84(1.18- \\
2.86), P=0.007\end{array}$ & $\begin{array}{l}1.01(0.63- \\
1.64), P=0.96\end{array}$ & 1 & $\begin{array}{l}1.16(0.77- \\
1.75), P=0.47\end{array}$ & $\begin{array}{l}1.13(0.71- \\
1.80), P=0.62\end{array}$ \\
\hline $\begin{array}{l}\text { Order } \\
\text { cohorts }\end{array}$ & 620 & 781 & 1160 & 1186 & 703 \\
\hline $\begin{array}{l}\text { No. of } \\
\text { deaths }\end{array}$ & $33(0.053)$ & $26(0.033)$ & $\begin{array}{l}31 \\
(0.027)\end{array}$ & $31(0.026)$ & $22(0.031)$ \\
\hline $\begin{array}{l}\text { Model } 1 \text { (HR, } \\
95 \% \mathrm{Cl})\end{array}$ & $\begin{array}{l}2.08(1.27- \\
3.39), P=0.003\end{array}$ & $\begin{array}{l}1.26(0.75- \\
2.13), P=0.38\end{array}$ & 1 & $\begin{array}{l}0.98(0.59- \\
1.61), P=0.93\end{array}$ & $\begin{array}{l}1.16(0.67- \\
2.00), P=0.59\end{array}$ \\
\hline $\begin{array}{l}\text { Model } 2(\mathrm{HR}, \\
95 \% \mathrm{Cl})\end{array}$ & $\begin{array}{l}1.78(1.08- \\
2.92), P=0.02\end{array}$ & $\begin{array}{l}1.18(0.70- \\
2.00), P=0.53\end{array}$ & 1 & $\begin{array}{l}1.01(0.61- \\
1.67), P=0.97\end{array}$ & $\begin{array}{l}1.34(0.77- \\
2.34), P=0.30\end{array}$ \\
\hline \multicolumn{6}{|c|}{ Ischaemic stroke } \\
\hline Cohort & 1134 & 1798 & 3366 & 4452 & 3228 \\
\hline $\begin{array}{l}\text { No. of } \\
\text { deaths }\end{array}$ & $22(0.019)$ & $11(0.006)$ & $\begin{array}{l}20 \\
(0.006)\end{array}$ & $22(0.005)$ & $11(0.003)$ \\
\hline $\begin{array}{l}\text { Model } 1(\mathrm{HR}, \\
95 \% \mathrm{Cl})\end{array}$ & $\begin{array}{l}3.30(1.80- \\
6.04), P<0.001\end{array}$ & $\begin{array}{l}1.02(0.49- \\
2.12), P=0.96\end{array}$ & 1 & $\begin{array}{l}0.83(0.46- \\
1.53), P=0.56\end{array}$ & $\begin{array}{l}0.57(0.28- \\
1.20), P=0.14\end{array}$ \\
\hline $\begin{array}{l}\text { Model } 2 \text { (HR, } \\
95 \% \mathrm{Cl})\end{array}$ & $\begin{array}{l}2.31(1.25- \\
4.26), P=0.008\end{array}$ & $\begin{array}{l}0.87(0.42- \\
1.82), P=0.71\end{array}$ & 1 & $\begin{array}{l}1.04(0.57- \\
1.92), P=0.89\end{array}$ & $\begin{array}{l}0.80(0.38- \\
1.70), P=0.56\end{array}$ \\
\hline $\begin{array}{l}\text { Order } \\
\text { cohort\$ }\end{array}$ & 607 & 764 & 1145 & 1167 & 689 \\
\hline $\begin{array}{l}\text { No. of } \\
\text { deaths }\end{array}$ & $20(0.033)$ & $9(0.012)$ & $\begin{array}{l}16 \\
(0.014)\end{array}$ & $12(0.010)$ & $8(0.012)$ \\
\hline
\end{tabular}

\$: Postmenopausal women born before 1940 were analyzed; model 1: a crude hazard ratio model without adjustments; model 2: a multivariate adjust model including age, diabetes, hypertension, dyslipidemia, smoking, alcohol drinking, physical activity, body mass index, self-rated health, education, job, family income, number of children and oral contraceptive pill use. 


\begin{tabular}{|c|c|c|c|c|c|}
\hline & \multicolumn{5}{|c|}{ Duration of reproductive years } \\
\hline & $\leq 28$ & $29-31$ & $32-34$ & $35-37$ & $\geq 38$ \\
\hline $\begin{array}{l}\text { Model } 1(\mathrm{HR}, \\
95 \% \mathrm{Cl})\end{array}$ & $\begin{array}{l}2.47(1.28- \\
4.77), P=0.007\end{array}$ & $\begin{array}{l}0.85(0.38- \\
1.93), P=0.70\end{array}$ & 1 & $\begin{array}{l}0.74(0.35- \\
1.55), P=0.42\end{array}$ & $\begin{array}{l}0.82(0.35- \\
1.91), P=0.64\end{array}$ \\
\hline $\begin{array}{l}\text { Model } 2 \text { (HR, } \\
95 \% \mathrm{Cl})\end{array}$ & $\begin{array}{l}2.22(1.14- \\
4.32), P=0.02\end{array}$ & $\begin{array}{l}0.83(0.37- \\
1.89), P=0.66\end{array}$ & 1 & $\begin{array}{l}0.77(0.36- \\
1.64), P=0.50\end{array}$ & $\begin{array}{l}0.97(0.41- \\
2.31), P=0.95\end{array}$ \\
\hline \multicolumn{6}{|c|}{$\begin{array}{l}\text { \$: Postmenopausal women born before } 1940 \text { were analyzed; model 1: a crude hazard ratio model } \\
\text { without adjustments; model 2: a multivariate adjust model including age, diabetes, hypertension, } \\
\text { dyslipidemia, smoking, alcohol drinking, physical activity, body mass index, self-rated health, } \\
\text { education, job, family income, number of children and oral contraceptive pill use. }\end{array}$} \\
\hline
\end{tabular}

\section{Discussion}

In this study, we found that late menarche, early menopause and fewer reproductive years were related to an increased risk of fatal stroke in Chinese postmenopausal women. The association was independent of age, diabetes, hypertension, dyslipidaemia, smoking, alcohol consumption, physical activity, body mass index, self-rated health, education, job, family income, number of children and oral contraceptive pill use.

Previous studies are inconsistent in terms of the association between age at menarche and stroke [8-12, $16-19,24,25]$. In a previous cohort study of 37,965 Japanese postmenopausal women, age $\geq 17$ years at menarche was associated with a low risk of mortality from stroke compared with age at menarche $\leq$ 13 years [19], and a cohort study of 66104 Korean women showed an association between risk of mortality from stroke and thrombotic stroke [25]. Our findings were similar in that late menarche was associated with an increased risk of fatal stroke occurrence. A U-shaped relationship between both early and late menarche and the increased risk of stroke incidence was observed in 1.2 million British women aged 50 to 64 years without prior heart disease, stroke, or cancer. This finding is the same as ours, wherein fatal stroke occurrence occurred in older women born before 1940 but not born after the 1960s in China [14]. The different associations between menarche and stroke (including stroke, ischaemic stroke and haemorrhagic stroke) need to be further explored in different ethnic populations.

It remains unclear why menarche is related to stroke events. We observed that menarche in relation to fatal stroke occurrence was independent of removal of the uterus, ovaries, or breast masses (benign or malignant) or use of hormone replacement therapy. This result suggests that the reproductive hormonal effect is not involved. The remaining associations, after further analysis of never smoking and additional adjustments for childhood passive smoking exposure, suggest that smoking is not an important factor. As in a previous study addressing early menarche in relation to metabolic syndrome and its components $[30,35]$, our results were independent of BMI, hypertension, diabetes, and other lifestyle and cardiovascular risk factors. Moreover, other unmeasured factors, including lower birth weight, higher body weight and weight gain in infancy and childhood, may be related to age at menarche and increase the risk of early menarche [36]. Additionally, in utero exposure and active physical activity during pregnancy [37] may be related to a modest delay in age at menarche among offspring. Anorexia, malnutrition and 
premenarchal intense exercise training are related to delayed menarche [38, 39]. Thus, a U-shaped association here may be due to unmeasured but unrelated genetic factors [40].

There is an inconsistent association between menopause and stroke $[10-13,15,19-23,26]$. Women aged 80 years or older, but not those aged $<80$ years, who were $<40$ years old at menopause had a 2.29 times higher stroke mortality risk than those aged $45-49$ years at menopause [13]. Here, a lower age at menopause (< 43 years) was associated with higher fatal stroke occurrence in older generations born before 1940 but not in those born in 1940 or later. However, other reports show inconsistent findings [19$21,23]$. Similar to other reports [17, 22], we found no significant associations between menopause and fatal ischaemic stroke or fatal haemorrhagic stroke, which may be due to the small number of stroke death cases.

Early menopause in relation to the risk of fatal stroke occurrence is due mainly to changes in endogenous steroid hormones, especially oestradiol, after menopause [41, 42]. Oestradiol should be a protective factor in stroke, although hormone replacement therapy does not play a beneficiary role [43, 44]. Here, we observed a similar association regardless of whether we excluded a series of factors or adjusted for lifestyle and cardiovascular risk factors.

The reproductive years constitute the interval between menarche and menopause. Most studies have reported that a low number of reproductive years is related to an increased fatal stroke or stroke incidence $[10,12,25-27]$, though there are contradictory reports $[8,19]$. Here, fewer reproductive years ( $\leq 28$ years) was associated with an increased fatal stroke and fatal ischaemic stroke occurrence, which is consistent with a Korean cohort study addressing an increased risk for developing stroke and thrombotic stroke [25] and a case-control study on nonembolic ischaemic stroke risk [12]. Similar associations for fatal stroke were reported only by three teams: one in China and two in the United States [10,26, 27]. An inconsistent relationship between reproductive years and fatal stroke occurrence was observed in China and Japan [8, 19].

In our study, a median follow-up of 12.0 years makes this a large, prospective design for a study of the general population in South China, and individuals completed a physical examination and questionnaire involving a total of 800 questions. Thus, the acquired information allows for systemic adjustments for additional potential confounding factors. However, there are limitations in this study. First, we obtained only the death information via record linkage with the GZCDC. The results of our study with death as the only outcome are obviously weakened because of the lack of analysis of other clinical outcomes of stroke events. Second, among a series of potential confounders, there may be some inaccuracies in the risk factors, such as self-reported age at menarche and menopause, from the multi-year recall [45-47]. Third, the subjects could not represent Chinese individuals due to the limitations involving the general population in South China in this study. Finally, the small number of deaths limited the strength of this study to address fatal stroke, especially fatal ischaemic stroke and fatal haemorrhagic stroke.

\section{Conclusion}


Age at menarche, age at menopause and number of reproductive years were related to the risk of fatal stroke occurrence. The burden of stroke may be increased in certain populations, and such women should be given more attention during the decade after menopause.

\section{Abbreviations}

HR

hazard ratio; Cl:confidence interval; GBCS:Guangzhou Biobank Cohort Study; GZCDC:Guangzhou Centers for Disease Control and Prevention; ICD:International Classification of Diseases; IVT:intravenous thrombolysis; IPAQ:International Physical Activity Questionnaire; OC:oral contraceptive

\section{Declarations}

\section{Acknowledgements}

The Guangzhou Biobank Cohort Study investigators included Guangzhou Twelfth People's Hospital: Weisen Zhang, Min Cao, Tong Zhu, Bin Liu, and Caoqiang Jiang (Co-PI); The University of Hong Kong: C.M. Schooling, S.M. McGhee, G.M. Leung, R. Fielding, and Taihing Lam (Co-PI); The University of Birmingham: P. Adab, G. Neil Thomas, and Karkeung Cheng (Co-PI).

\section{Authors' contributions}

FZ contributed to the study design. ZBH and ZXL contributed to data collection and analysis. FZ wrote the manuscript. All authors reviewed the manuscript.

\section{Authors' information}

Zhi-Bing Hu: 18022868581@163.com

Ze-Xiong Lu: 1158103839@qq.com

Feng Zhu: chifengzhu@hotmail.com

\section{Funding}

This work was supported by the Guangzhou Science and Technology Bureau, Guangzhou, China (201704030132, 202102080467) and Guangdong Provincial Medical Science Research Foundation, Guangdong, China (A2021124).

\section{Availability of data and materials}

Data used in this analysis are available from the corresponding author on reasonable request.

\section{Consent to publication}


Not applicable.

\section{Ethics approval and consent to participate}

Our study was approved by the Guangzhou Medical Ethics Committee of the Chinese Medical Association, and all participants provided written informed consent before participation in the GBCS. All methods in this study were performed in accordance with the Declaration of Helsinki.

\section{Competing interest}

No conflicts of interest to declare.

\section{References}

1. Group GBDNDC: Global, regional, and national burden of neurological disorders during 1990-2015: a systematic analysis for the Global Burden of Disease Study 2015. The Lancet Neurology 2017, 16(11):877-897.

2. Zhou M, Wang H, Zhu J, Chen W, Wang L, Liu S, Li Y, Wang L, Liu Y, Yin P et al: Cause-specific mortality for 240 causes in China during 1990-2013: a systematic subnational analysis for the Global Burden of Disease Study 2013. Lancet 2016, 387(10015):251-272.

3. McCullough LD, Mirza MA, Xu Y, Bentivegna K, Steffens EB, Ritzel R, Liu F: Stroke sensitivity in the aged: sex chromosome complement vs. gonadal hormones. Aging 2016, 8(7):1432-1441.

4. Leening MJ, Ferket BS, Steyerberg EW, Kavousi M, Deckers JW, Nieboer D, Heeringa J, Portegies ML, Hofman A, Ikram MA et al: Sex differences in lifetime risk and first manifestation of cardiovascular disease: prospective population based cohort study. Bmj 2014, 349:g5992.

5. Wieberdink RG, Ikram MA, Hofman A, Koudstaal PJ, Breteler MM: Trends in stroke incidence rates and stroke risk factors in Rotterdam, the Netherlands from 1990 to 2008. European journal of epidemiology 2012, 27(4):287-295.

6. Spaander FH, Zinkstok SM, Baharoglu IM, Gensicke H, Polymeris A, Traenka C, Hametner C, Ringleb P, Curtze S, Martinez-Majander $\mathrm{N}$ et al: Sex Differences and Functional Outcome After Intravenous Thrombolysis. Stroke 2017, 48(3):699-703.

7. Soriano-Reixach MM, Vivanco-Hidalgo RM, Ois A, Rodriguez-Campello A, Roquer J: Interaction of Sex and Diabetes on Outcome After Ischemic Stroke. Frontiers in neurology 2018, 9:250.

8. Wu X, Cai H, Kallianpur A, Gao YT, Yang G, Chow WH, Li HL, Zheng W, Shu XO: Age at menarche and natural menopause and number of reproductive years in association with mortality: results from a median follow-up of 11.2 years among $\mathbf{3 1 , 9 5 5}$ naturally menopausal Chinese women. PloS one 2014, 9(8):e103673.

9. Jacobsen BK, Heuch I, Kvale G: Age at natural menopause and stroke mortality: cohort study with 3561 stroke deaths during 37-year follow-up. Stroke 2004, 35(7):1548-1551. 
10. Ley SH, Li Y, Tobias DK, Manson JE, Rosner B, Hu FB, Rexrode KM: Duration of Reproductive Life Span, Age at Menarche, and Age at Menopause Are Associated With Risk of Cardiovascular Disease in Women. Journal of the American Heart Association 2017, 6(11).

11. Murakami K, Metoki H, Satoh M, Asayama K, Hosaka M, Matsuda A, Inoue R, Tsubota-Utsugi M, Murakami T, Nomura K et al: Menstrual Factors and Stroke Incidence in Japanese Postmenopausal Women: The Ohasama Study. Neuroepidemiology 2016, 47(2):109-116.

12. Alonso de Lecinana M, Egido JA, Fernandez C, Martinez-Vila E, Santos S, Morales A, Martinez E, Pareja A, Alvarez-Sabin J, Casado I et al: Risk of ischemic stroke and lifetime estrogen exposure. Neurology 2007, 68(1):33-38.

13. Hong JS, Yi SW, Kang HC, Jee SH, Kang HG, Bayasgalan G, Ohrr H: Age at menopause and causespecific mortality in South Korean women: Kangwha Cohort Study. Maturitas 2007, 56(4):411-419.

14. Yang L, Li L, Millwood IY, Peters SAE, Chen Y, Guo Y, Bian Z, Chen X, Chen L, Feng S et al: Age at menarche and risk of major cardiovascular diseases: Evidence of birth cohort effects from a prospective study of 300,000 Chinese women. International journal of cardiology $2017,227: 497-$ 502.

15. Liberale L, Montecucco F, Bonaventura A, Casetta I, Seraceni S, Trentini A, Padroni M, Dallegri F, Fainardi E, Carbone F: Monocyte count at onset predicts poststroke outcomes during a 90-day followup. Eur J Clin Invest 2017, 47(10):702-710.

16. Charalampopoulos D, McLoughlin A, Elks CE, Ong KK: Age at menarche and risks of all-cause and cardiovascular death: a systematic review and meta-analysis. American journal of epidemiology 2014, 180(1):29-40.

17. Gallagher LG, Davis LB, Ray RM, Psaty BM, Gao DL, Checkoway H, Thomas DB: Reproductive history and mortality from cardiovascular disease among women textile workers in Shanghai, China. International journal of epidemiology 2011, 40(6):1510-1518.

18. Mueller NT, Odegaard AO, Gross MD, Koh WP, Yuan JM, Pereira MA: Age at menarche and cardiovascular disease mortality in Singaporean Chinese women: the Singapore Chinese Health Study. Annals of epidemiology 2012, 22(10):717-722.

19. Cui R, Iso H, Toyoshima H, Date C, Yamamoto A, Kikuchi S, Kondo T, Watanabe Y, Koizumi A, Inaba Y et al: Relationships of age at menarche and menopause, and reproductive year with mortality from cardiovascular disease in Japanese postmenopausal women: the JACC study. Journal of epidemiology 2006, 16(5):177-184.

20. Mondul AM, Rodriguez C, Jacobs EJ, Calle EE: Age at natural menopause and cause-specific mortality. American journal of epidemiology 2005, 162(11):1089-1097.

21. Jacobsen BK, Oda K, Knutsen SF, Fraser GE: Age at menarche, total mortality and mortality from ischaemic heart disease and stroke: the Adventist Health Study, 1976-88. International journal of epidemiology 2009, 38(1):245-252.

22. Hu FB, Grodstein F, Hennekens CH, Colditz GA, Johnson M, Manson JE, Rosner B, Stampfer MJ: Age at natural menopause and risk of cardiovascular disease. Archives of internal medicine 1999, 
159(10):1061-1066.

23. de Kleijn MJ, van der Schouw YT, Verbeek AL, Peeters PH, Banga JD, van der Graaf Y: Endogenous estrogen exposure and cardiovascular mortality risk in postmenopausal women. American journal of epidemiology 2002, 155(4):339-345.

24. Canoy D, Beral V, Balkwill A, Wright FL, Kroll ME, Reeves GK, Green J, Cairns BJ, Million Women Study C: Age at menarche and risks of coronary heart and other vascular diseases in a large UK cohort. Circulation 2015, 131(3):237-244.

25. Jung KJ, Kim MR, Yun YD, Kim HC, Jee SH: Duration of ovarian hormone exposure and atherosclerotic cardiovascular disease in Korean women: the Korean Heart Study. Menopause 2016, 23(1):60-66.

26. Yang L, Lin L, Kartsonaki C, Guo Y, Chen Y, Bian Z, Xie K, Jin D, Li L, Lv J et al: Menopause Characteristics, Total Reproductive Years, and Risk of Cardiovascular Disease Among Chinese Women. Circulation Cardiovascular quality and outcomes 2017, 10(11).

27. Mansoor H, Elgendy IY, Segal R, Hartzema A: Duration of Reproductive Years and the Risk of Cardiovascular and Cerebrovascular Events in Older Women: Insights from the National Health and Nutrition Examination Survey. Journal of women's health 2017, 26(10):1047-1052.

28. Jiang C, Thomas GN, Lam TH, Schooling CM, Zhang W, Lao X, Adab P, Liu B, Leung GM, Cheng KK: Cohort profile: The Guangzhou Biobank Cohort Study, a Guangzhou-Hong Kong-Birmingham collaboration. Int J Epidemio/ 2006, 35(4):844-852.

29. Deng HB, Macfarlane DJ, Thomas GN, Lao XQ, Jiang CQ, Cheng KK, Lam TH: Reliability and validity of the IPAQ-Chinese: the Guangzhou Biobank Cohort study. Medicine and science in sports and exercise 2008, 40(2):303-307.

30. Heys M, Schooling CM, Jiang C, Cowling BJ, Lao X, Zhang W, Cheng KK, Adab P, Thomas GN, Lam TH et al: Age of menarche and the metabolic syndrome in China. Epidemiology 2007, 18(6):740746.

31. Lewington S, Li L, Murugasen S, Hong LS, Yang L, Guo Y, Bian Z, Collins R, Chen J, He H et al: Temporal trends of main reproductive characteristics in ten urban and rural regions of China: the China Kadoorie biobank study of $\mathbf{3 0 0} \mathbf{0 0 0}$ women. International journal of epidemiology 2014, 43(4):1252-1262.

32. Yang S, Jin Y, He Y, Jiang C, Cheng KK, Zhang W, Lam TH: Childhood Passive Smoking Exposure and Age at Menarche in Chinese Women Who Had Never Smoked: The Guangzhou Biobank Cohort Study. PloS one 2015, 10(7):e0130429.

33. Ertunc D, Tok EC, Aytan $H$, Gozukara YM: Passive smoking is associated with lower age at menopause. Climacteric: the journal of the International Menopause Society 2015, 18(1):47-52.

34. Hou L, Han W, Jiang J, Liu B, Wu Y, Zou X, Xue F, Chen Y, Zhang B, Pang H et al: Passive smoking and stroke in men and women: a national population-based case-control study in China. Scientific reports 2017, 7:45542. 
35. Chang CJ, Lai MM, Lin CC, Liu CS, Li TC, Li Cl, Lin WY: Age at menarche and its association with the metabolic syndrome in Taiwan. Obesity research \& clinical practice 2016, 10 Suppl 1:S26-S34.

36. Juul F, Chang VW, Brar P, Parekh N: Birth weight, early life weight gain and age at menarche: a systematic review of longitudinal studies. Obesity reviews: an official journal of the International Association for the Study of Obesity 2017, 18(11):1272-1288.

37. Colbert LH, Graubard BI, Michels KB, Willett WC, Forman MR: Physical activity during pregnancy and age at menarche of the daughter. Cancer epidemiology, biomarkers \& prevention: a publication of the American Association for Cancer Research, cosponsored by the American Society of Preventive Oncology 2008, 17(10):2656-2662.

38. Rees M: Menarche when and why? Lancet 1993, 342(8884):1375-1376.

39. Rosetta L: Female reproductive dysfunction and intense physical training. Oxford reviews of reproductive biology 1993, 15:113-141.

40. He C, Kraft P, Chasman DI, Buring JE, Chen C, Hankinson SE, Pare G, Chanock S, Ridker PM, Hunter DJ: A large-scale candidate gene association study of age at menarche and age at natural menopause. Human genetics 2010, 128(5):515-527.

41. Cai J, Li J, Chen S, Meng J, Ren R, Li M: Early post-haemorrhagic stroke testosterone and oestradiol levels and long-term risk of death. Brain injury 2017, 31(3):401-405.

42. Guo JM, Shu H, Wang L, Xu JJ, Niu XC, Zhang L: SIRT1-dependent AMPK pathway in the protection of estrogen against ischemic brain injury. CNS neuroscience \& therapeutics 2017, 23(4):360-369.

43. Brass LM: Hormone replacement therapy and stroke: clinical trials review. Stroke 2004, 35(11 Suppl 1):2644-2647.

44. Bath PM, Gray LJ: Association between hormone replacement therapy and subsequent stroke: a meta-analysis. Bmj 2005, 330(7487):342.

45. Must A, Phillips SM, Naumova EN, Blum M, Harris S, Dawson-Hughes B, Rand WM: Recall of early menstrual history and menarcheal body size: after 30 years, how well do women remember? American journal of epidemiology 2002, 155(7):672-679.

46. Lundblad MW, Jacobsen BK: The reproducibility of self-reported age at menarche: The Tromso Study. BMC women's health 2017, 17(1):62.

47. Colditz GA, Stampfer MJ, Willett WC, Stason WB, Rosner B, Hennekens CH, Speizer FE: Reproducibility and validity of self-reported menopausal status in a prospective cohort study. American journal of epidemiology 1987, 126(2):319-325.

\section{Figures}




20343 women participants at postmenopausal
recruited for baseline of the Guangzhou Biobank
Cohort Study

\begin{tabular}{|c|c|c|}
\hline \multirow{3}{*}{$\begin{array}{l}1334 \text { participants with } \\
\text { incomplete information on } \\
\text { diabetes, hypertension, } \\
\text { dyslipidemia, smoking, alcohol } \\
\text { drinking, physical activity, } \\
\text { body mass index, self-rated } \\
\text { health, education, job, family } \\
\text { income, number of children } \\
\text { and oral contraceptive pill use }\end{array}$} & & $\begin{array}{l}1741 \text { participants with heart } \\
\text { disease and peripheral vascular } \\
\text { disease history }\end{array}$ \\
\hline & & $\begin{array}{l}\text { 157participants with previous } \\
\text { history of stroke }\end{array}$ \\
\hline & & $\begin{array}{l}244 \text { participants loss to follow- } \\
\text { up with unknown vital status }\end{array}$ \\
\hline
\end{tabular}

16504 participants at baseline were recorded in this study (from September 2003 to February 2008)

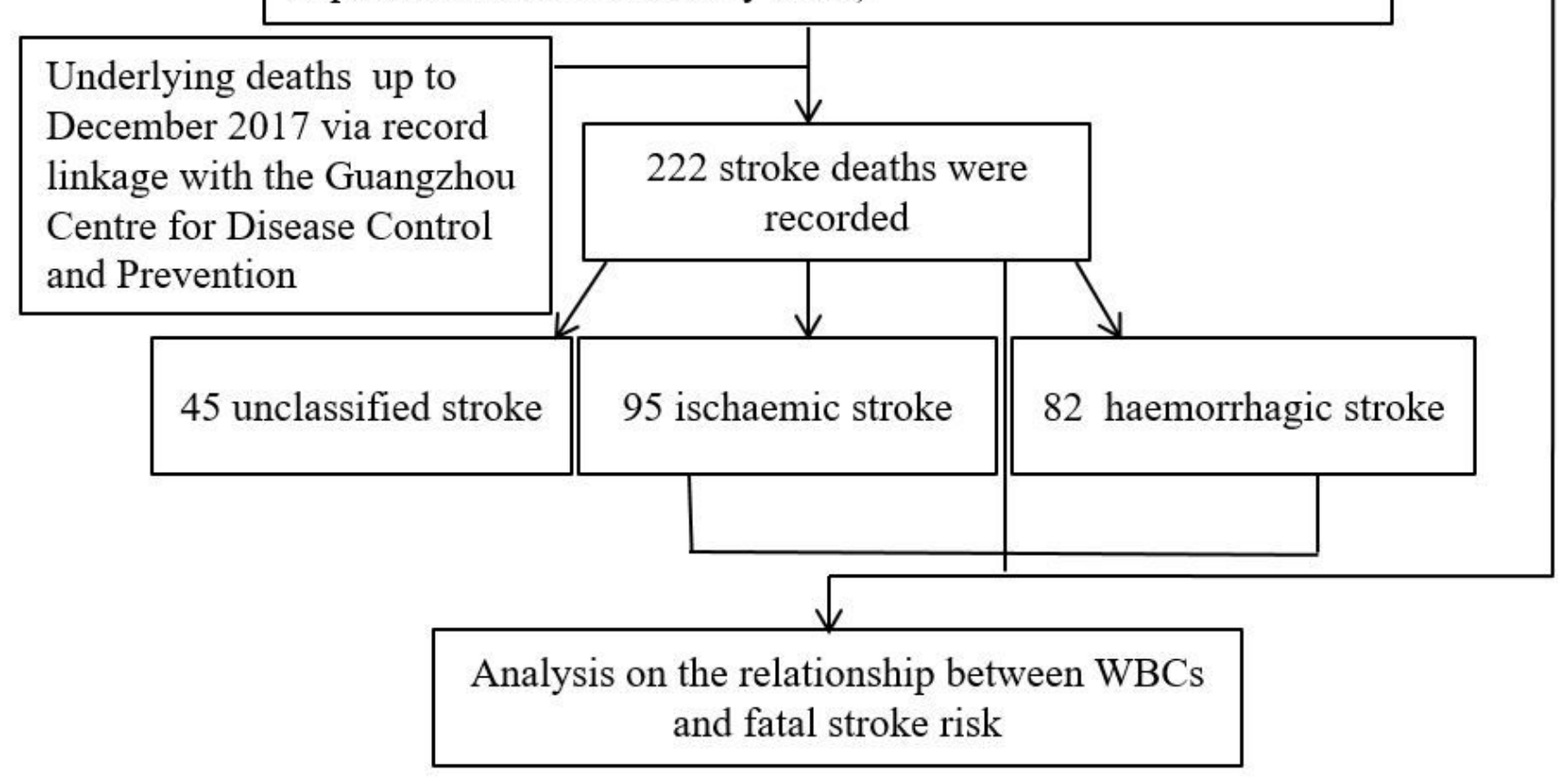

Figure 1

Flow diagram of participants selected for the analysis of this study 